YITP-SB-12-20

\title{
Form factors and complete spectrum of XXX antiperiodic higher spin chains by quantum separation of variables
}

\author{
G. Niccoli $1^{1}$
}

\begin{abstract}
The antiperiodic transfer matrix associated to higher spin representations of the rational 6-vertex Yang-Baxter algebra is analyzed by generalizing the approach introduced recently in [1], for the cyclic representations, in [2], for the spin-1/2 highest weight representations, and in [3], for the spin 1/2 representations of the reflection algebra. Here, we derive the complete characterization of the transfer matrix spectrum and we prove its simplicity in the framework of Sklyanin's quantum separation of variables (SOV). Then, the characterization of local operators by Sklyanin's quantum separate variables and the expression of the scalar products of separates states by determinant formulae allow to compute the form factors of the local spin operators by one determinant formulae similar to the scalar product ones. Finally, let us comment that these results represent the SOV analogous in the antiperiodic higher spin XXX quantum chains of the results obtained for the periodic chains in [4] in the framework of the algebraic Bethe ansatz.
\end{abstract}

\footnotetext{
${ }^{1}$ YITP, Stony Brook University, New York 11794-3840, USA, niccoli@ max2.physics.sunysb.edu
} 


\section{Contents}

1 Introduction 3

2 Antiperiodic 6-vertex models 4

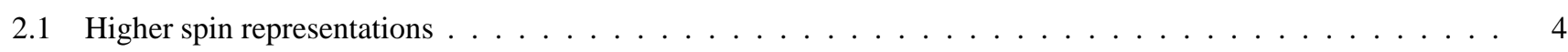

2.1.1 Yang-Baxter algebra representations on vector and covector spaces $\ldots \ldots \ldots \ldots$

2.1.2 Rational 6-vertex higher spin transfer matrices $\ldots \ldots \ldots \ldots \ldots \ldots \ldots \ldots \ldots \ldots \ldots$

2.2 Antiperiodic 6 -vertex quantum integrable higher spin chains $\ldots \ldots \ldots \ldots \ldots \ldots$

2.2 .1 Fusion procedure for 6 -vertex representations $\ldots \ldots \ldots \ldots \ldots \ldots \ldots$

2.2.2 Antiperiodic higher $\operatorname{spin} \mathrm{XXX}$ quantum chains $\ldots \ldots \ldots \ldots \ldots \ldots$

3 SOV-representations $r$

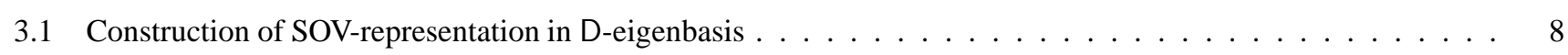

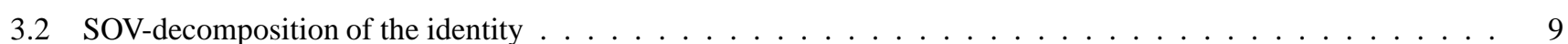

$4 \quad \bar{T}$-spectrum characterization by SOV

$5 \bar{T}$-decomposition of the identity $\quad 12$

6 Reconstruction of local spin generators $\quad 13$

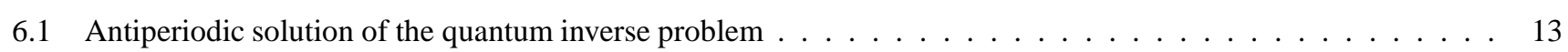

7 Form factors of local operators

8 Conclusion and outlook

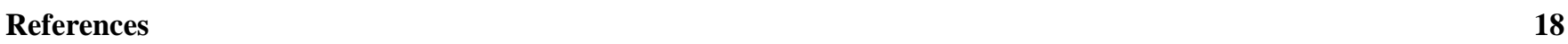




\section{Introduction}

The lattice quantum integrable models associated by the quantum inverse scattering method (QISM) [5]-[18] to the representations of the rational 6-vertex Yang-Baxter algebra on higher spin- $s$ quantum chains ( $s$ any positive half-integer) with antiperiodic boundary conditions are analyzed. Under the homogeneous limit, such analysis allows to describe the spin- $s$ XXX quantum chain with antiperiodic boundary conditions. Then, it is worth recalling that under periodic boundary conditions 2 these quantum models have been investigated by using the algebraic Bethe ansatz 3 (ABA) and results are known both for the spectrum characterization and for the correlation functions [31, 4]. In the papers [32, 33], the analysis of the spectrum under general toroidal boundary conditions has been developed thanks to the extension to these quantum models of the ABA method. It is also relevant to comment that similarly, by using the Baxter's gauge transformation technique [34], the spectrum of the open XXX quantum spin chains with general non-diagonal integrable boundary conditions has been analyzed by ABA in [35, 36] and also by the functional version of Sklyanin's quantum separation of variables (SOV) [37]-[39] in [40, 41].

Here, we show how to apply an approach based on Sklyanin's SOV to the special case of antiperiodic boundary condition:55, which allows to achieve the complete characterization of the spectrum and the computation of the matrix elements of local operators on the transfer matrix eigenstates. It is natural to consider the analysis here presented as the generalization to the SOV-framework of the Lyon group method [44]-[57] implemented in the ABA framework6. This approach has been first developed 7 in [1, 77] for the lattice quantum sine-Gordon model [6, 18] and for the $\tau_{2}$-mode 8 [82]. In particular, in [1, 77] the reconstruction of local operators by quantum separate variables and one determinant formulae for the scalar products of separate states 9 have been derived and used to compute matrix elements of local operators in determinant form. Further key quantum integrable models have been analyzed by this approach getting the same type of universal results. In [2] the antiperiodid 10 XXZ spin $1 / 2$ quantum chain [85]-[93] has been considered while for the spin $1 / 2$ representations of the reflection algebra [94]-[102] with non-diagonal boundaries the SOV setup has been implemented in [3] and there also the matrix elements of some string of local operators have been computed.

Let us finally comment that our interest toward the SOV method of Sklyanin is due to the fact that it allows to overcome several problems which affect others methods like for example the coordinate Bethe ansatz [86], [34] and [103], the Baxter Q-operator method [34], the algebraic Bethe ansatz [5]-[6], the analytic Bethe ansatz [104]-[105]. Indeed, SOV applies for a large class of integrable quantum models to which others Bethe ansatz methods do not apply; both the eigenvalues and the eigenstates are constructed and under simple conditions these spectrum characterizations are complete. Moreover, in all the models analyzed in the series of papers [61]-[64], [2], [3] and [106] in the SOV framework the non-degeneracy of the transfer matrix spectrum has been proven.

\footnotetext{
${ }^{2}$ See [19]-[25], [15] and [26] for the first studies of the integrable spin-s XXZ quantum spin chains. The spin-1 Hamiltonian was first given in [19]; Bethe ansatz equations for the spin- $s$ models with periodic boundary conditions were obtained and studied in [22] 25] 27], see also [28]-[30].

${ }^{3}$ See [5]-[6] and reference therein.

${ }^{4}$ In fact, the analysis developed in these papers also generalizes the (nested) ABA for the integrable quantum models associated to rational higher rank Yang-Baxter algebras for these general boundary conditions.

${ }^{5}$ Let us recall that in [42] has been developed the analysis by the functional SOV of the related but more general spin-boson model introduced and first analyzed by ABA in 43 .

${ }^{6}$ See [58]-60] for the extension of this method and of the corresponding results to the open spin $1 / 2$ quantum chains with diagonal boundary conditions.

${ }^{7}$ Let us comment that these papers use as required setup the series of papers [61-64 where the complete spectrum has been characterized for the lattice quantum sine-Gordon model and for the $\tau_{2}$-model and the chiral Potts model [65]-[76], respectively.

${ }^{8}$ See the series of works [78]-81] for previous analysis by SOV method of the $\tau_{2}$-model.

${ }^{9}$ See Section 5 for the definition of these states in our current model.

${ }^{10}$ Let us comment that previous results for this model under antiperiodic boundary condition were based on the Baxter Q-operator [83] and the functional separation of variables of Sklyanin developed first in [38] for the XXX spin chain and later in [84] for the XXZ spin chain.
} 


\section{Antiperiodic 6-vertex models}

\subsection{Higher spin representations}

Let $S_{n}^{ \pm}$and $S_{n}^{z}$ be the generators of the $\operatorname{sl}(2)$ algebra:

$$
\left[S^{z}, S^{ \pm}\right]= \pm S^{ \pm},\left[S^{+}, S^{-}\right]=2 S^{z}
$$

and let $\mathrm{R}^{\left(s_{n}\right)} \simeq \mathbb{C}^{2 s_{n}+1}$ be linear spaces (local quantum spaces) of dimension $\left(2 s_{n}+1\right)$ with $2 s_{n} \in \mathbb{Z}^{>0}$. A spin- $s_{n}$ representation of the $\operatorname{sl}(2)$ algebra is associated to any linear space $\mathrm{R}^{\left(s_{n}\right)}$ by defining:

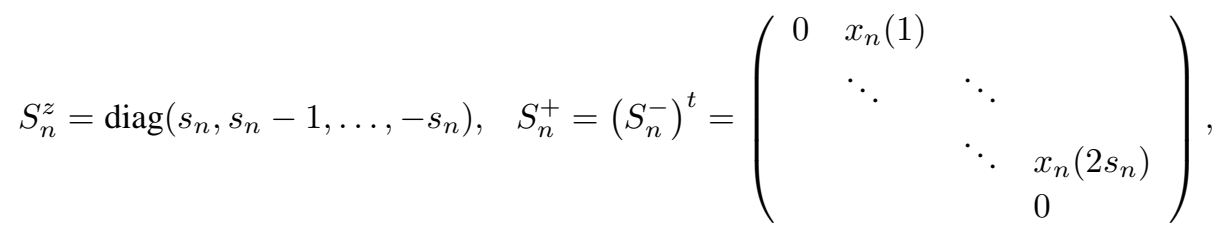

where $x_{n}(j) \equiv \sqrt{j\left(2 s_{n}+1-j\right)}$. Then, to each local quantum space $\mathrm{R}^{\left(s_{n}\right)}$ is associated a so-called Lax operator:

$$
\mathrm{L}_{0 n}^{\left(1 / 2, s_{n}\right)}(\lambda) \equiv\left(\begin{array}{cc}
\lambda+\eta\left(1 / 2+S_{n}^{z}\right) & \eta S_{n}^{-} \\
\eta S_{n}^{+} & \lambda+\eta\left(1 / 2-S_{n}^{z}\right)
\end{array}\right)_{0} \in \operatorname{End}\left(V_{0}^{(1 / 2)} \otimes V_{n}^{\left(s_{n}\right)}\right),
$$

which satisfies the Yang-Baxter equation:

$$
R_{12}(\lambda-\mu) \mathrm{L}_{1 n}^{\left(1 / 2, s_{n}\right)}(\lambda) \mathrm{L}_{2 n}^{\left(1 / 2, s_{n}\right)}(\mu)=\mathrm{L}_{2 n}^{\left(1 / 2, s_{n}\right)}(\mu) \mathrm{L}_{1 n}^{\left(1 / 2, s_{n}\right)}(\lambda) R_{12}(\lambda-\mu),
$$

w.r.t. the rational 6-vertex R-matrix:

$$
R_{12}(\lambda)=\mathrm{L}_{12}^{(1 / 2,1 / 2)}(\lambda) \equiv\left(\begin{array}{cccc}
\lambda+\eta & 0 & 0 & 0 \\
0 & \lambda & \eta & 0 \\
0 & \eta & \lambda & 0 \\
0 & 0 & 0 & \lambda+\eta
\end{array}\right)
$$

Now, we are in the position to define the so-called monodromy matrix:

$$
\mathrm{M}_{0}^{(1 / 2)}(\lambda) \equiv\left(\begin{array}{ll}
\mathrm{A}(\lambda) & \mathrm{B}(\lambda) \\
\mathrm{C}(\lambda) & \mathrm{D}(\lambda)
\end{array}\right) \equiv \mathrm{L}_{0 \mathrm{~N}}^{\left(1 / 2, s_{\mathrm{N}}\right)}\left(\lambda-\eta_{\mathrm{N}}\right) \cdots \mathrm{L}_{01}^{\left(1 / 2, s_{1}\right)}\left(\lambda-\eta_{1}\right) \in \operatorname{End}\left(\mathrm{R}_{0}^{(1 / 2)} \otimes_{n=1}^{\mathrm{N}} \mathrm{R}_{n}^{\left(s_{n}\right)}\right),
$$

where the $\eta_{n}$ are the so-called inhomogeneities parameters. Then the monodromy matrix $\mathrm{M}_{0}^{(1 / 2)}(\lambda)$ is itself solution of the Yang-Baxter equation:

$$
R_{12}(\lambda-\mu) \mathrm{M}_{1}^{(1 / 2)}(\lambda) \mathrm{M}_{2}^{(1 / 2)}(\mu)=\mathrm{M}_{2}^{(1 / 2)}(\mu) \mathrm{M}_{1}^{(1 / 2)}(\lambda) R_{12}(\lambda-\mu),
$$

w.r.t. the rational 6-vertex R-matrix.

\subsubsection{Yang-Baxter algebra representations on vector and covector spaces}

Let $|k, n\rangle$ be a vector in $\mathrm{R}_{n}^{\left(s_{n}\right)}$ characterized by:

$$
S_{n}^{z}|k, n\rangle=\left(k-s_{n}\right)|k, n\rangle, \quad k \in\left\{0,1, \ldots, 2 s_{n}\right\},
$$

i.e. the set of $|k, n\rangle$ defines a $S_{n}^{z}$-eigenbasis of the local space $\mathrm{R}_{n}^{\left(s_{n}\right)}$, and let us denote with $\mathrm{L}_{n}^{\left(s_{n}\right)}$ the linear space dual of $\mathbf{R}_{n}^{\left(s_{n}\right)}$ and with $\langle k, n|$ a covector defined by:

$$
\left\langle k, n \mid k^{\prime}, n\right\rangle \equiv \delta_{k, k^{\prime}} \quad \forall k, k^{\prime} \in\left\{0, \ldots, 2 s_{n}\right\}
$$


i.e. the covectors $\langle k, n|$ define the $S_{n}^{z}$-eigenbasis in the dual linear space $\mathrm{L}_{n}$. We can naturally introduce a scalar product w.r.t. the covector-vector basis by setting:

$$
\left(|k, n\rangle,\left|k^{\prime}, n\right\rangle\right) \equiv\left\langle k, n \mid k^{\prime}, n\right\rangle .
$$

In the left (covectors) and right (vectors) linear spaces:

$$
\mathcal{L}_{\mathrm{N}} \equiv \otimes_{n=1}^{\mathrm{N}} \mathrm{L}_{n}^{\left(s_{n}\right)}, \quad \mathcal{R}_{\mathrm{N}} \equiv \otimes_{n=1}^{\mathrm{N}} \mathrm{R}_{n}^{\left(s_{n}\right)},
$$

the representations of the local $s l(2)$ generators induce left and right spin- $\left\{s_{1}, \ldots, s_{\mathrm{N}}\right\}$ representations of dimension $d_{\mathrm{N}} \equiv$ $\prod_{n=1}^{\mathrm{N}}\left(2 s_{n}+1\right)$ with $\mathrm{N}$ inhomogeneities of the rational 6-vertex Yang-Baxter algebra; i.e. the quadratic algebra defined by the set of commutation relations of the monodromy matrix elements $\mathrm{A}(\lambda), \mathrm{B}(\lambda), \mathrm{C}(\lambda)$ and $\mathrm{D}(\lambda)$ encoded in the Yang-Baxter equation satisfied by $\mathrm{M}_{0}^{(1 / 2)}(\lambda)$.

\subsubsection{Rational 6-vertex higher spin transfer matrices}

Let us remark that the rational 6-vertex $R$-matrix satisfies the following $G L(2, \mathbb{C})$ symmetry:

$$
R_{12}(\lambda) W_{1} \otimes W_{2}=W_{2} \otimes W_{1} R_{12}(\lambda),
$$

where $W$ is any invertible $2 \times 2$ matrix. Then, we have that for any $W$ we can define a monodromy matrix:

$$
\mathrm{M}_{0}^{(1 / 2, W)}(\lambda) \equiv W_{0} \mathrm{M}_{0}^{(1 / 2)}(\lambda),
$$

which is a solution of the Yang-Baxter equations (2.4) w.r.t. the same rational 6-vertex $R$-matrix. This imply that the transfer matrix:

$$
\mathbf{T}^{(W)}(\lambda)=\operatorname{tr}_{0}\left[W_{0} \mathbf{M}_{0}^{(1 / 2)}(\lambda)\right],
$$

defines a one-parameter family of commuting operators. Moreover, let us recall that the quantum determinant:

$$
\operatorname{det}_{q} \mathrm{M}_{0}^{(1 / 2, W)}(\lambda) \equiv \operatorname{det} W \operatorname{det}_{q} \mathrm{M}_{0}^{(1 / 2)}(\lambda)
$$

where

$$
\underset{q}{\operatorname{det}} \mathrm{M}_{0}^{\left(1 / 2, \sigma^{x}\right)}(\lambda) \equiv \mathrm{B}(\lambda) \mathrm{C}(\lambda / q)-\mathrm{A}(\lambda) \mathrm{D}(\lambda / q)
$$

is a central elemen 11 of the Yang-Baxter algebra (2.4) which explicit reads:

$$
\operatorname{det}_{q} \mathrm{M}_{0}^{\left(1 / 2, \sigma^{x}\right)}(\lambda) \equiv \operatorname{det}_{q} \overline{\mathrm{M}}^{(1 / 2)}(x)=a(\lambda) d(\lambda-\eta),
$$

where 12 :

$$
a(\lambda)=-\prod_{n=1}^{\mathrm{N}}\left(\lambda-\eta_{n}^{-}+s_{n} \eta\right), \quad d(\lambda)=\prod_{n=1}^{\mathrm{N}}\left(\lambda-\eta_{n}^{-}-s_{n} \eta\right),
$$

and we have used the notation $\lambda^{ \pm} \equiv \lambda \pm \eta / 2$.

In the following, we solve the spectral problem for the quantum integrable models characterized in the framework of the quantum inverse scattering method by the following antiperiodic transfer matrix:

$$
\overline{\mathbf{T}}(\lambda) \equiv \mathrm{B}(\lambda)+\mathrm{C}(\lambda)=\mathrm{T}^{\left(W=\sigma^{x}\right)}(\lambda) .
$$

Lemma 2.1. I) If $\eta \in i \mathbb{R}$ and $\left\{\eta_{1}, \ldots, \eta_{\mathrm{N}}\right\} \in \mathbb{R}^{\mathrm{N}}, \overline{\mathrm{T}}(\lambda)$ is a one parameter family of normal operators and:

$$
i \overline{\mathbf{T}}(\lambda)
$$

is self-adjoint for any $\lambda \in \mathbb{C}$ such that $\lambda-\lambda^{*}+\eta=0$.

II) If $\eta \in \mathbb{R}$ and $\left\{\eta_{1}, \ldots, \eta_{\mathrm{N}}\right\} \in(i \mathbb{R})^{\mathrm{N}}, \overline{\mathrm{T}}(\lambda)$ is a one parameter family of normal operators and:

$$
i^{\mathrm{e}_{\mathrm{N}}} \overline{\mathrm{T}}(\lambda),
$$

where $\mathrm{e}_{\mathrm{N}}=\{1$ for $N$ even, 0 for $N$ odd $\}$, is self-adjoint for any $\lambda \in \mathbb{C}$ such that $\lambda+\lambda^{*}+\eta=0$.

\footnotetext{
${ }^{11}$ See [107] and [108] for an historical note.

${ }^{12}$ Note that $a(\lambda)$ has zeros at $\lambda=\eta_{n}^{-}-s_{n} \eta$, and $d(\lambda)$ has zeros at $\lambda=\eta_{n}^{-}+s_{n} \eta$ for all $j=1, \ldots, \mathrm{N}$.
} 
Proof. I) $\mathrm{L}_{0 n}^{\left(1 / 2, s_{n}\right)}(\lambda)$ satisfies the following Hermitian conjugation property:

$$
\mathrm{L}_{0 n}^{\left(1 / 2, s_{n}\right)}(\lambda)^{\dagger} \equiv \sigma_{0}^{y} \mathrm{~L}_{0 n}^{\left(1 / 2, s_{n}\right)}\left(\lambda^{*}-\eta\right) \sigma_{0}^{y},
$$

as it can be verified by direct computations. Here $\dagger$ implements the transposition on the local quantum space $n$ and the complex conjugation, then it holds:

$$
\mathrm{M}(\lambda)^{\dagger} \equiv\left(\begin{array}{cc}
\mathrm{A}^{\dagger}(\lambda) & \mathrm{B}^{\dagger}(\lambda) \\
\mathrm{C}^{\dagger}(\lambda) & \mathrm{D}^{\dagger}(\lambda)
\end{array}\right)=\left(\begin{array}{cc}
\mathrm{D}\left(\lambda^{*}-\eta\right) & -\mathrm{C}\left(\lambda^{*}-\eta\right) \\
-\mathrm{B}\left(\lambda^{*}-\eta\right) & \mathrm{A}\left(\lambda^{*}-\eta\right)
\end{array}\right)
$$

for $\left\{\eta_{1}, \ldots, \eta_{\mathrm{N}}\right\} \in \mathbb{R}^{\mathrm{N}}$ and so $\overline{\mathbf{T}}(\lambda)$ is normal for any $\lambda \in \mathbb{C}$ and the statements in I) follow.

II) It is simple to observe that:

$$
\mathrm{L}_{0 n}^{\left(1 / 2, s_{n}\right)}(\lambda)^{\dagger} \equiv-\sigma_{0}^{y} \mathrm{~L}_{0 n}^{\left(1 / 2, s_{n}\right)}\left(-\left(\lambda^{*}+\eta\right)\right) \sigma_{0}^{y}
$$

then, it holds:

$$
\mathrm{M}(\lambda)^{\dagger} \equiv\left(\begin{array}{cc}
\mathrm{A}^{\dagger}(\lambda) & \mathrm{B}^{\dagger}(\lambda) \\
\mathrm{C}^{\dagger}(\lambda) & \mathrm{D}^{\dagger}(\lambda)
\end{array}\right)=(-1)^{\mathrm{N}}\left(\begin{array}{cc}
\mathrm{D}\left(-\left(\lambda^{*}+\eta\right)\right) & -\mathrm{C}\left(-\left(\lambda^{*}+\eta\right)\right) \\
-\mathrm{B}\left(-\left(\lambda^{*}+\eta\right)\right) & \mathrm{A}\left(-\left(\lambda^{*}+\eta\right)\right)
\end{array}\right),
$$

for $\left\{\eta_{1}, \ldots, \eta_{\mathrm{N}}\right\} \in(i \mathbb{R})^{\mathrm{N}}$ and so $\overline{\mathbf{T}}(\lambda)$ is normal for any $\lambda \in \mathbb{C}$ and the statements in II) follow.

\subsection{Antiperiodic 6-vertex quantum integrable higher spin chains}

\subsubsection{Fusion procedure for 6-vertex representations}

The fusion procedure was first developed in [26] for the case of the rational 6-vertex representations of the type analyzed here and later in [27] for the trigonometric ones. Our interest in the fusion procedure is related to its use to reconstruct local operators in terms of the Yang-Baxter algebra generators and the fused transfer matrix. This result was first derived in the case of the periodic transfer matrix $\left(W=\mathbb{I}_{2 \times 2}\right)$ in [45]; here we will extend that result in the case of the antiperiodic $\left(W=\sigma^{x}\right)$ transfer matrices.

The fusion procedure can be used to construct monodromy matrices with auxiliary spaces of dimension higher than 2 starting from the one with 2-dimensional auxiliary space. We illustrate this procedure in the antiperiodic case following a presentation similar to that of [109]. Let us remark that the following commutation relations hold:

$$
\left[\mathrm{L}_{0 a}^{\left(1 / 2, s_{a}\right)}(\lambda), \sigma_{0}^{x} \otimes \Sigma_{a}^{x}\right]=0, \quad \forall a \in\{1, \ldots, \mathrm{N}\}
$$

where $\Sigma_{a}^{x}$ is the $\left(2 s_{a}+1\right) \times\left(2 s_{a}+1\right)$ matrix with elements all zeros except those along the antidiagonal which are 1 . The above property is a consequence of the commutation relations:

$$
S_{a}^{ \pm} \Sigma_{a}^{x}=\Sigma_{a}^{x} S_{a}^{\mp}, \quad S_{a}^{z} \Sigma_{a}^{x}=-\Sigma_{a}^{x} S_{a}^{z}
$$

where $S_{a}^{ \pm}$and $S_{a}^{z}$ are the generators of the spin- $\left(2 s_{a}+1\right)$ representation of $s l(2)$. Form (2.26), we have also:

$$
\left[\mathrm{M}_{0}^{(1 / 2)}(\lambda), \sigma_{0}^{x} \otimes \Sigma^{x}\right]=0, \quad \forall a \in\{1, \ldots, \mathrm{N}\}
$$

where:

$$
\Sigma^{x} \equiv \otimes_{a=1}^{\mathrm{N}} \Sigma_{a}^{x}
$$

Let us now define the antiperiodic monodromy matrices:

$$
\overline{\mathrm{M}}_{0}^{(1 / 2)}(\lambda) \equiv \sigma_{0}^{x} \mathrm{M}_{0}^{(1 / 2)}(\lambda) \in \operatorname{End}\left(\mathrm{R}_{0}^{(1 / 2)} \otimes \mathcal{R}_{\mathrm{N}}\right), \quad \overline{\mathrm{M}}_{a}^{\left(s_{a}\right)}(\lambda) \equiv \Sigma_{a}^{x} \mathrm{M}_{a}^{\left(s_{a}\right)}(\lambda) \in \operatorname{End}\left(\mathrm{R}_{a}^{\left(s_{a}\right)} \otimes \mathcal{R}_{\mathrm{N}}\right),
$$

and denoted with $\mathbf{R}_{\langle 12\rangle}^{(s)}$ and $\mathbf{R}_{(12)}^{(s-1)}$ the linear spaces of dimension $2 s+1$ and $2 s-1$ defined by the following decomposition

$$
\mathbf{R}_{1}^{\left(s-\frac{1}{2}\right)} \otimes \mathbf{R}_{2}^{\left(\frac{1}{2}\right)} \simeq \mathbf{R}_{\langle 12\rangle}^{(s)} \oplus \mathbf{R}_{(12)}^{(s-1)},
$$


of the tensor product of $\mathbf{R}_{2}^{\left(\frac{1}{2}\right)}$ and $\mathbf{R}_{1}^{\left(s-\frac{1}{2}\right)}$, linear spaces of dimension 2 and $2 s$, respectively. This tensor product decomposition allows to define the operator:

$$
P_{12}=P_{\langle 12\rangle}^{+} \oplus P_{(12)}^{-},
$$

as the direct sum of the projector $P_{\langle 12\rangle}^{+}$in $\mathbf{R}_{\langle 12\rangle}^{(s)}$ and the projector $P_{(12)}^{-}$in $\mathbf{R}_{(12)}^{(s-1)}$. Then the fusion of the monodromy matrices $\overline{\mathrm{M}}_{a}^{\left(s_{a}\right)}(\lambda)$ has the same form as for the periodic case and it reads:

$$
P_{12} \overline{\mathrm{M}}_{1}^{(1 / 2)}\left(\lambda^{-}+s \eta\right) \overline{\mathrm{M}}_{2}^{(s-1 / 2)}\left(\lambda^{-}\right) P_{12}=\left(\begin{array}{cc}
\overline{\mathrm{M}}_{\langle 12\rangle}^{(s)}(\lambda) & 0 \\
* & \overline{\mathrm{M}}_{(12)}^{(s-1)}(\lambda-\eta) \operatorname{det}_{q} \overline{\mathrm{M}}^{(1 / 2)}(\lambda+(s-1) \eta)
\end{array}\right),
$$

where now the quantum determinant of the antiperiodic monodromy matrix $\overline{\mathrm{M}}^{(1 / 2)}(\lambda)$ appears. Note that to any (higher) monodromy matrix $\overline{\mathrm{M}}_{0}^{(s)}(\lambda)$ we can associate the one-parameter family of higher transfer matrix:

$$
\overline{\mathrm{T}}^{(s)}(\lambda)=\operatorname{tr}_{0} \overline{\mathrm{M}}_{0}^{(s)}(\lambda) \in \operatorname{End}\left(\mathcal{R}_{\mathrm{N}}\right) \forall \lambda \in \mathbb{C},
$$

which define commuting families of operators:

$$
\left[\overline{\mathbf{T}}^{\left(s_{1}\right)}(\lambda), \overline{\mathbf{T}}^{\left(s_{2}\right)}(\mu)\right]=0 \quad \forall 2 s_{1}, 2 s_{2} \in \mathbb{Z}^{>0} .
$$

The formula (2.33) implies in particular the following recursion relations:

$$
\overline{\mathbf{T}}^{(s)}(\lambda)=\overline{\mathbf{T}}^{(1 / 2)}\left(\lambda^{-}+s \eta\right) \overline{\mathbf{T}}^{(s-1 / 2)}\left(\lambda^{-}\right)-\operatorname{det}_{q} \overline{\mathrm{M}}^{(1 / 2)}(\lambda+(s-1) \eta) \overline{\mathbf{T}}^{(s-1)}(\lambda-\eta),
$$

for antiperiodic higher transfer matrices.

\subsubsection{Antiperiodic higher spin XXX quantum chains}

It is worth pointing out that the analysis of the antiperiodic transfer matrix $\overline{\mathbf{T}}^{(1 / 2)}(\lambda)$ allows in particular to describe the XXX higher spin- $s$ quantum chains in the special case of the homogeneous limit $\left(\eta_{n} \rightarrow 0\right)$ and under homogeneous spin- $s$ representations $\left(s_{n}=s\right.$ for any $\left.n \in\{1, \ldots, \mathrm{N}\}\right)$. Indeed, the Hamiltonian of the XXX spin- $s$ quantum chain is obtain by logarithmic derivative of the transfer matrix associated to the fundamental monodromy matrix $\overline{\mathrm{M}}^{(s)}(\lambda)$ :

$$
H=\left.\overline{\mathbf{T}}^{(s)}(\lambda)^{-1} \frac{d}{d \lambda} \overline{\mathbf{T}}^{(s)}(\lambda)\right|_{\lambda=0},
$$

with the following boundary conditions:

$$
S_{\mathrm{N}+1}^{z}=-S_{1}^{z}, \quad S_{\mathrm{N}+1}^{ \pm}=S_{1}^{\mp} .
$$

Note that the transfer matrix $\overline{\mathrm{T}}^{(s)}(\lambda)$ used to construct the XXX spin- $s$ Hamiltonian is obtained in terms of the transfer matrix $\overline{\mathbf{T}}(\lambda)$ by using the recursion relations (2.36). This point together with the simplicity of the spectrum of the transfer matrix $\overline{\mathbf{T}}(\lambda)$ (which we will show in the following) implies that it is enough to characterize the spectrum of this last transfer matrix to have in particular the solution of the XXX spin-s quantum chain.

\section{SOV-representations}

A separation of variable (SOV) representation [37, 38, 39] for the $\overline{\mathrm{T}}$-spectral problem is associated to a representation for which the commutative family of operators $\mathrm{D}(\lambda)$ (or $\mathrm{A}(\lambda)$ ) is diagonal and with simple spectrum.

Theorem 3.1. If the inhomogeneities $\left\{\eta_{1}, \ldots, \eta_{\mathrm{N}}\right\} \in \mathbb{C}^{\mathrm{N}}$ satisfy the conditions:

$$
\eta_{a} \neq \eta_{b} \bmod \eta \quad \forall a \neq b \in\{1, \ldots, \mathrm{N}\}
$$

then $\mathrm{D}(\lambda)$ and $\mathrm{A}(\lambda)$ are diagonalizable and with simple spectrum and the $\overline{\mathrm{T}}$-spectral problem admits separate variable representations.

In the next subsection we construct explicitly the D-eigenbasis in this way proving the Theorem 3.1 


\subsection{Construction of SOV-representation in D-eigenbasis}

Let

$$
\langle 0| \equiv \otimes_{n=1}^{\mathrm{N}}\langle 1, n| \quad \text { and }|0\rangle \equiv \otimes_{n=1}^{\mathrm{N}}|1, n\rangle,
$$

be the left (covector) and right (vector) references states, where:

$$
\left\langle 1, n\left|=(1,0, \ldots, 0)_{1,2 s_{n}+1}, \quad\right| 1, n\right\rangle=\left(\begin{array}{c}
1 \\
0 \\
\vdots \\
0
\end{array}\right)_{2 s_{n}+1,1},
$$

then:

Theorem 3.2. I) Left $\mathrm{D}(\lambda)$ SOV-representations If (3.1) are verified, the states $\langle\boldsymbol{h}| \equiv\left\langle h_{1}, \ldots, h_{\mathrm{N}}\right|$, defined by:

$$
\langle\boldsymbol{h}| \equiv \frac{1}{\mathrm{~N}}\langle 0| \prod_{n=1}^{N} \prod_{k_{n}=0}^{h_{n}-1} \frac{C\left(\eta_{n}^{\left(k_{n}\right)}\right)}{d\left(\eta_{n}^{\left(k_{n}+1\right)}\right)},
$$

where

$$
\mathrm{N}=\prod_{1 \leq b<a \leq \mathrm{N}}\left(\eta_{a}^{(0)}-\eta_{b}^{(0)}\right)^{1 / 2},
$$

$h_{n} \in\left\{0, \ldots, 2 s_{n}\right\}$ for all the $n \in\{1, \ldots, \mathrm{N}\}$ and:

$$
\eta_{n}^{\left(k_{n}\right)} \equiv \eta_{n}^{-}+\left(s_{n}-k_{n}\right) \eta
$$

define a $\mathrm{D}$-eigenbasis of $\mathcal{L}_{\mathrm{N}}$ :

$$
\langle\boldsymbol{h}| \mathrm{D}(\lambda)=d_{\boldsymbol{h}}(\lambda)\langle\boldsymbol{h}|,
$$

where:

$$
d_{\boldsymbol{h}}(\lambda) \equiv \prod_{n=1}^{\mathrm{N}}\left(\lambda-\eta_{n}^{\left(h_{n}\right)}\right) \quad \text { and } \boldsymbol{h} \equiv\left(h_{1}, \ldots, h_{\mathrm{N}}\right)
$$

Moreover it holds:

$$
\begin{aligned}
\langle\boldsymbol{h}| \mathrm{C}(\lambda) & =\sum_{a=1}^{\mathrm{N}} \prod_{b \neq a} \frac{\lambda-\eta_{b}^{\left(h_{b}\right)}}{\eta_{a}^{\left(h_{a}\right)}-\eta_{b}^{\left(h_{b}\right)}} d\left(\eta_{a}^{\left(h_{a}+1-\beta_{h_{a}}\right)}\right)\langle\boldsymbol{h}| T_{a}^{+}, \\
\langle\boldsymbol{h}| \mathrm{B}(\lambda) & =\sum_{a=1}^{\mathrm{N}} \prod_{b \neq a} \frac{\lambda-\eta_{b}^{\left(h_{b}\right)}}{\eta_{a}^{\left(h_{a}\right)}-\eta_{b}^{\left(h_{b}\right)}} a\left(\eta_{a}^{\left(h_{a}-1+\alpha_{h_{a}}\right)}\right)\langle\boldsymbol{h}| T_{a}^{-},
\end{aligned}
$$

where:

$$
\alpha_{h_{a}} \equiv\left(2 s_{a}+1\right) \delta_{h_{a}, 0}, \quad \beta_{h_{a}} \equiv\left(2 s_{a}+1\right) \delta_{h_{a}, 2 s_{a}}, \quad\left\langle h_{1}, \ldots, h_{a}, \ldots, h_{\mathrm{N}}\right| T_{a}^{ \pm}=\left\langle h_{1}, \ldots, h_{a} \pm 1, \ldots, h_{\mathrm{N}}\right| .
$$

Finally, $\mathrm{A}(\lambda)$ is uniquely defined by the quantum determinant relation.

II) Right $\mathrm{D}(\lambda)$ SOV-representations If (3.1) are verified, the states $|\boldsymbol{h}\rangle \equiv\left|h_{1}, \ldots, h_{\mathrm{N}}\right\rangle$, defined by:

$$
|\boldsymbol{h}\rangle \equiv \frac{1}{\mathrm{~N}} \prod_{n=1}^{\mathrm{N}} \prod_{k_{n}=0}^{h_{n}-1} \frac{B\left(\eta_{n}^{\left(k_{n}\right)}\right)}{a\left(\eta_{n}^{\left(k_{n}\right)}\right)}|0\rangle,
$$

where $h_{n} \in\left\{0, \ldots, 2 s_{n}\right\}$ for all the $n \in\{1, \ldots, N\}$, define a D-eigenbasis of $\mathcal{R}_{N}$ :

$$
\mathrm{D}(\lambda)|\boldsymbol{h}\rangle=d_{\boldsymbol{h}}(\lambda)|\boldsymbol{h}\rangle .
$$


Moreover, it holds:

$$
\begin{aligned}
\mathrm{C}(\lambda)|\boldsymbol{h}\rangle & =\sum_{a=1}^{\mathrm{N}} T_{a}|\boldsymbol{h}\rangle \prod_{b \neq a} \frac{\lambda-\eta_{b}^{\left(h_{b}\right)}}{\eta_{a}^{\left(h_{a}\right)}-\eta_{b}^{\left(h_{b}\right)}} d\left(\eta_{a}^{\left(h_{a}\right)}\right), \\
\mathrm{B}(\lambda)|\boldsymbol{h}\rangle & =\sum_{a=1}^{\mathrm{N}} T_{a}^{+}|\boldsymbol{h}\rangle \prod_{b \neq a} \frac{\lambda-\eta_{b}^{\left(h_{b}\right)}}{\eta_{a}^{\left(h_{a}\right)}-\eta_{b}^{\left(h_{b}\right)}} a\left(\eta_{a}^{\left(h_{a}\right)}\right),
\end{aligned}
$$

where:

$$
T_{a}^{ \pm}\left|h_{1}, \ldots, h_{a}, \ldots, h_{\mathrm{N}}\right\rangle=\left|h_{1}, \ldots, h_{a} \pm 1, \ldots, h_{\mathrm{N}}\right\rangle .
$$

Finally, $\mathrm{A}(\lambda)$ is uniquely defined by the quantum determinant relation.

Proof. The proof of the theorem is based on Yang-Baxter commutation relations and on the fact that the left and right references states are D-eigenstates:

$$
\langle 0| \mathrm{A}(\lambda)=a(\lambda)\langle 0|, \quad\langle 0| \mathrm{D}(\lambda)=d(\lambda)\langle 0|, \quad\langle 0| \mathrm{B}(\lambda)=\underline{0}, \quad\langle 0| \mathrm{C}(\lambda) \neq \underline{0},
$$

and

$$
\mathrm{A}(\lambda)|0\rangle=a(\lambda)|0\rangle, \quad \mathrm{D}(\lambda)|0\rangle=d(\lambda)|0\rangle, \quad \mathrm{C}(\lambda)|0\rangle=\underline{0}, \quad \mathrm{~B}(\lambda)|0\rangle \neq \underline{0} .
$$

Indeed, to prove that (3.4) and (3.12) are left and right eigenstates of $D(\lambda)$ as stated in (3.7) and (3.13), we have just to repeat the standard computations in algebraic Bethe ansatz [13] as done in the proof of Theorem 3.2 of [2].

Note that representations of the type (3.9)-(3.10) and (3.14) $-(\sqrt{3.15})$ for the generators of the 6-vertex Yang-Baxter algebra are also derived from the original representations by the change of basis associated to the factorizing $F$-matrices [110]. These matrices give explicit representations of the Drinfel'd's twist of quasi-triangular quasi-Hopf algebras [111]-[113] and their connection with Sklyanin's quantum separation of variables was pointed out in [114] providing the factorizing $F$-matrices for general Yangian $Y(\operatorname{sl}(2))$.

\subsection{SOV-decomposition of the identity}

The following proposition holds:

Proposition 3.1. Let $\langle\boldsymbol{h}|$ be the generic left D-eigenstate and $|\boldsymbol{k}\rangle$ be the generic right $\mathrm{D}$-eigenstate, then the action of the covector $\langle\boldsymbol{h}|$ on the vector $|\boldsymbol{k}\rangle$ reads:

$$
\langle\boldsymbol{h} \mid \boldsymbol{k}\rangle=\prod_{c=1}^{\mathrm{N}} \delta_{h_{c}, k_{c}} \prod_{1 \leq b<a \leq \mathrm{N}} \frac{1}{\eta_{a}^{\left(h_{a}\right)}-\eta_{b}^{\left(h_{b}\right)}} .
$$

Proof. The identity:

$$
\left(d_{\mathbf{h}}(\lambda)-d_{\mathbf{k}}(\lambda)\right)\langle\mathbf{h} \mid \mathbf{k}\rangle=0 \quad \forall \lambda \in \mathbb{C},
$$

obtained by computing $\langle\mathbf{h}|\mathrm{D}(\lambda)| \mathbf{k}\rangle$ implies:

$$
\langle\mathbf{h} \mid \mathbf{k}\rangle=0 \quad \forall \mathbf{h} \neq \mathbf{k} \in\left\{0, \ldots, 2 s_{1}\right\} \times \ldots . \times\left\{0, \ldots, 2 s_{\mathrm{N}}\right\},
$$

then we have just to compute $\langle\mathbf{h} \mid \mathbf{h}\rangle$. In order to do so we compute $\theta_{a} \equiv\left\langle h_{1}, \ldots, h_{a}-1, \ldots, h_{\mathrm{N}}\left|C\left(\eta_{a}^{\left(h_{a}-1\right)}\right)\right| h_{1}, \ldots, h_{a}, \ldots, h_{\mathrm{N}}\right\rangle$, where $1 \leq h_{a} \leq 2 s_{a}$ and $a \in\{1, \ldots, \mathrm{N}\}$. In our SOV-representation the left action of $C\left(\eta_{a}^{\left(h_{a}-1\right)}\right)$ reads:

$$
\theta_{a}=d\left(\eta_{a}^{\left(h_{a}\right)}\right)\left\langle h_{1}, \ldots, h_{a}, \ldots, h_{\mathrm{N}} \mid h_{1}, \ldots, h_{a}, \ldots, h_{\mathrm{N}}\right\rangle, \text { being } \beta_{h_{a}-1}=0 \text { for } 1 \leq h_{a} \leq 2 s_{a},
$$

while the right action plus the identity (3.21) imply:

$$
\theta_{a}=\prod_{b \neq a, b=1}^{\mathrm{N}} \frac{\eta_{a}^{\left(h_{a}-1\right)}-\eta_{b}^{\left(h_{b}\right)}}{\eta_{a}^{\left(h_{a}\right)}-\eta_{b}^{\left(h_{b}\right)}} d\left(\eta_{a}^{\left(h_{a}\right)}\right)\left\langle h_{1}, \ldots, h_{a}-1, \ldots, h_{\mathrm{N}} \mid h_{1}, \ldots, h_{a}-1, \ldots, h_{\mathrm{N}}\right\rangle,
$$


and so:

$$
\frac{\left\langle h_{1}, \ldots, h_{a}, \ldots, h_{\mathrm{N}} \mid h_{1}, \ldots, h_{a}, \ldots, h_{\mathrm{N}}\right\rangle}{\left\langle h_{1}, \ldots, h_{a}-1, \ldots, h_{\mathrm{N}} \mid h_{1}, \ldots, h_{a}-1, \ldots, h_{\mathrm{N}}\right\rangle}=\prod_{b \neq a, b=1}^{\mathrm{N}} \frac{\eta_{a}^{\left(h_{a}-1\right)}-\eta_{b}^{\left(h_{b}\right)}}{\eta_{a}^{\left(h_{a}\right)}-\eta_{b}^{\left(h_{b}\right)}}
$$

This result implies:

$$
\frac{\left\langle h_{1}, \ldots, h_{\mathrm{N}} \mid h_{1}, \ldots, h_{\mathrm{N}}\right\rangle}{\langle 0 \mid 0\rangle / \mathrm{N}^{2}}=\prod_{1 \leq b<a \leq \mathrm{N}} \frac{\eta_{a}^{(0)}-\eta_{b}^{(0)}}{\eta_{a}^{\left(h_{a}\right)}-\eta_{b}^{\left(h_{b}\right)}},
$$

then the definition (3.5) of the normalization $\mathrm{N}$ and the fact that:

$$
\langle 0 \mid 0\rangle=1,
$$

imply (3.19).

From the previous result and from the fact that $\mathrm{D}(\lambda)$ is diagonalizable and with simple spectrum we get the following decomposition of the identity $\mathbb{I}$ :

$$
\mathbb{I} \equiv \sum_{h_{1}=0}^{2 s_{1}} \ldots \sum_{h_{\mathrm{N}}=0}^{2 s_{\mathrm{N}}} \prod_{1 \leq b<a \leq \mathrm{N}}\left(\eta_{a}^{\left(h_{a}\right)}-\eta_{b}^{\left(h_{b}\right)}\right)\left|h_{1}, \ldots, h_{\mathrm{N}}\right\rangle\left\langle h_{1}, \ldots, h_{\mathrm{N}}\right| .
$$

\section{$4 \overline{\mathrm{T}}$-spectrum characterization by SOV}

Let

$$
\mathbb{C}_{\text {even }}[\lambda]_{\mathrm{N}-1} \text { for } \mathrm{N} \text { odd, } \mathbb{C}_{\text {odd }}[\lambda]_{\mathrm{N}-1} \text { for } \mathrm{N} \text { even, }
$$

be the linear spaces in the field $\mathbb{C}$ of the polynomials of degree $\mathrm{N}-1$ in the variable $\lambda$ even or odd as stated in the subscript, then, the set of the eigenvalue functions $t(\lambda)$ of $\overline{\mathrm{T}}(\lambda), \Sigma_{\overline{\mathrm{T}}}$ is contained in (4.1) and moreover it holds:

Theorem 4.1. Under the conditions (3.1), the spectrum of $\overline{\mathrm{T}}(\lambda)$ is simple and $\Sigma_{\overline{\overline{\mathrm{T}}}}$ coincides with the set of solutions of the discrete system of equations:

$$
\operatorname{det}_{2 s_{n}+1} D_{n}=0, \quad \forall n \in\{1, \ldots, \mathrm{N}\},
$$

in (4.1). Here, $D_{n}$ is the $\left(2 s_{n}+1\right) \times\left(2 s_{n}+1\right)$ tridiagonal matrix:

$$
D_{n} \equiv\left(\begin{array}{cccccc}
t\left(\eta_{n}^{(0)}\right) & -a\left(\eta_{n}^{(0)}\right) & 0 \ldots & & 0 & 0 \\
-d\left(\eta_{n}^{(1)}\right) & t\left(\eta_{n}^{(1)}\right) & -a\left(\eta_{n}^{(1)}\right) \ldots & & 0 & 0 \\
0 & \ddots & & & & \\
\vdots & & \ddots & & & \\
\vdots & & 0 \ldots & -d\left(\eta_{n}^{\left(2 s_{n}-1\right)}\right) & t\left(\eta_{n}^{\left(2 s_{n}-1\right)}\right) & -a\left(\eta_{n}^{\left(2 s_{n}-1\right)}\right) \\
0 & \ldots & 0 \ldots & 0 & -d\left(\eta_{n}^{\left(2 s_{n}\right)}\right) & t\left(\eta_{n}^{\left(2 s_{n}\right)}\right)
\end{array}\right)
$$

I) Up to an overall normalization, the right $\overline{\bar{\top}}$-eigenstate corresponding to $t(\lambda) \in \Sigma_{\overline{\bar{T}}}$ reads:

$$
|t\rangle=\sum_{h_{1}=0}^{2 s_{1}} \ldots \sum_{h_{\mathrm{N}}=0}^{2 s_{\mathrm{N}}} \prod_{a=1}^{\mathrm{N}} Q_{t}\left(\eta_{a}^{\left(h_{a}\right)}\right) \prod_{1 \leq b<a \leq \mathrm{N}}\left(\eta_{a}^{\left(h_{a}\right)}-\eta_{b}^{\left(h_{b}\right)}\right)\left|h_{1}, \ldots, h_{\mathrm{N}}\right\rangle,
$$

where:

$$
\begin{aligned}
Q_{t}\left(\eta_{a}^{\left(h_{a}+1\right)}\right) & =\frac{t\left(\eta_{a}^{\left(h_{a}\right)}\right)}{d\left(\eta_{a}^{\left(h_{a}+1\right)}\right)} Q_{t}\left(\eta_{a}^{\left(h_{a}\right)}\right)-\frac{a\left(\eta_{a}^{\left(h_{a}-1\right)}\right)}{d\left(\eta_{a}^{\left(h_{a}+1\right)}\right)} Q_{t}\left(\eta_{a}^{\left(h_{a}-1\right)}\right), \forall h_{a} \in\left\{1, \ldots, 2 s_{a}-1\right\}, \\
Q_{t}\left(\eta_{a}^{(1)}\right) & =Q_{t}\left(\eta_{a}^{(0)}\right) t\left(\eta_{a}^{(0)}\right) / d\left(\eta_{a}^{(1)}\right) .
\end{aligned}
$$


II) Up to an overall normalization, the left $\overline{\mathrm{T}}$-eigenstate corresponding to $t(\lambda) \in \Sigma_{\overline{\mathrm{T}}}$ reads:

$$
\langle t|=\sum_{h_{1}=0}^{2 s_{1}} \ldots \sum_{h_{\mathrm{N}}=0}^{2 s_{\mathrm{N}}} \prod_{a=1}^{\mathrm{N}} \bar{Q}_{t}\left(\eta_{a}^{\left(h_{a}\right)}\right) \prod_{1 \leq b<a \leq \mathrm{N}}\left(\eta_{a}^{\left(h_{a}\right)}-\eta_{b}^{\left(h_{b}\right)}\right)\left\langle h_{1}, \ldots, h_{\mathrm{N}}\right|,
$$

where:

$$
\begin{aligned}
\bar{Q}_{t}\left(\eta_{a}^{\left(h_{a}+1\right)}\right) & =\frac{t\left(\eta_{a}^{\left(h_{a}\right)}\right)}{a\left(\eta_{a}^{\left(h_{a}\right)}\right)} \bar{Q}_{t}\left(\eta_{a}^{\left(h_{a}\right)}\right)-\frac{d\left(\eta_{a}^{\left(h_{a}\right)}\right)}{a\left(\eta_{a}^{\left(h_{a}\right)}\right)} \bar{Q}_{t}\left(\eta_{a}^{\left(h_{a}-1\right)}\right), \quad \forall h_{a} \in\left\{1, \ldots, 2 s_{a}-1\right\} \\
\bar{Q}_{t}\left(\eta_{a}^{(1)}\right) & =\bar{Q}_{t}\left(\eta_{a}^{(0)}\right) t\left(\eta_{a}^{(0)}\right) / a\left(\eta_{a}^{(0)}\right)
\end{aligned}
$$

Proof. The wave-functions:

$$
\Psi_{t}(\mathbf{h}) \equiv\langle t \mid \mathbf{h}\rangle
$$

which are the coefficients of $\langle t|, \overline{\mathrm{T}}$-eigenstate corresponding to the eigenvalue $t(\lambda) \in \Sigma_{\overline{\mathrm{T}}}$, in the SOV-decomposition of the identity (3.27), satisfy the following discrete system of $d_{\mathrm{N}} \equiv \prod_{n=1}^{\mathrm{N}}\left(2 s_{n}+1\right)$ Baxter-like equations:

$$
t\left(\eta_{n}^{\left(h_{n}\right)}\right) \Psi_{t}(\mathbf{h})=a\left(\eta_{n}^{\left(h_{n}\right)}\right) \Psi_{t}\left(\mathbf{T}_{n}^{+}(\mathbf{h})\right)+d\left(\eta_{n}^{\left(h_{n}\right)}\right) \Psi_{t}\left(\mathbf{T}_{n}^{-}(\mathbf{h})\right),
$$

for any $n \in\{1, \ldots, N\}$ and $\mathbf{h} \in \otimes_{n=1}^{N}\left\{0, \ldots, 2 s_{n}\right\}$, where:

$$
\mathrm{T}_{n}^{ \pm}(\mathbf{h}) \equiv\left(h_{1}, \ldots, h_{n} \pm 1, \ldots, h_{\mathrm{N}}\right) .
$$

Now observing that:

$$
a\left(\eta_{n}^{\left(2 s_{n}\right)}\right)=d\left(\eta_{n}^{(0)}\right)=0
$$

we can rephrase the previous system by the homogeneous system of equations:

$$
D_{n}\left(\begin{array}{l}
\Psi_{t}\left(\cdots, h_{n}=0, \cdots\right) \\
\Psi_{t}\left(\cdots, h_{n}=1, \cdots\right) \\
\vdots \\
\vdots \\
\Psi_{t}\left(\cdots, h_{n}=2 s_{n}, \cdots\right)
\end{array}\right)_{\left(2 s_{n}+1\right) \times 1}=\left(\begin{array}{l}
0 \\
\vdots \\
\vdots \\
\vdots \\
0
\end{array}\right)_{\left(2 s_{n}+1\right) \times 1} \quad \forall n \in\{1, \ldots, \mathrm{N}\}
$$

From $t(\lambda) \in \Sigma_{\overline{\mathrm{T}}}$ follows that the determinants of the $\left(2 s_{n}+1\right) \times\left(2 s_{n}+1\right)$ matrix $D_{n}$ must be zero for any $n \in\{1, \ldots, N\}$, i.e. we get the system of equations (4.2). Let $\operatorname{det}\left(D_{n}\right)_{i, j}$ be the minor obtained by eliminating the row $i$ and the column $j$ from the matrix $D_{n}$, then being:

$$
\operatorname{det}\left(D_{n}\right)_{1,2 s_{n}+1}=\prod_{h_{n}=1}^{2 s_{n}} d\left(\eta_{n}^{\left(h_{n}\right)}\right) \neq 0,
$$

the matrices $D_{n}$ have rank $2 s_{n}$ and then the solution of (4.14) is unique up to an overall normalization. Then for any $t(\lambda) \in \Sigma_{\overline{\bar{T}}}$ there exist (up to normalization) one and only one $\overline{\mathrm{T}}$-eigenstate $\langle t|$ characterized by:

$$
\frac{\Psi_{t}\left(h_{1}, \ldots, h_{n}+1, \ldots, h_{1}\right)}{\Psi_{t}\left(h_{1}, \ldots, h_{n}, \ldots, h_{1}\right)}=\frac{\bar{Q}_{t}\left(\eta_{n}^{\left(h_{n}+1\right)}\right)}{\bar{Q}_{t}\left(\eta_{n}^{\left(h_{n}\right)}\right)},
$$

for any $n \in\{1, \ldots, N\}, h_{n} \in\left\{0, \ldots, 2 s_{n}-1\right\}$ and $h_{m \neq n} \in\left\{0, \ldots, 2 s_{m}\right\}$ as the equations (4.8)-(4.9) fix uniquely the ratios on the r.h.s. of the above equations; this proves the simplicity of the $\overline{\mathrm{T}}$-spectrum.

Vice versa, let $t(\lambda)$ be a solution of (4.2) in (4.1), then the state $|t\rangle$ constructed by (4.7)-(4.9) satisfies:

$$
\left\langle t\left|\overline{\mathbf{T}}\left(\eta_{n}^{\left(h_{n}\right)}\right)\right| h_{1}, \ldots, h_{\mathrm{N}}\right\rangle=t\left(\eta_{n}^{\left(h_{n}\right)}\right)\left\langle t \mid h_{1}, \ldots, h_{\mathrm{N}}\right\rangle \forall n \in\{1, \ldots, \mathrm{N}\}
$$

for any D-eigenstate $\left\langle h_{1}, \ldots, h_{\mathrm{N}}\right|$. This implies:

$$
\left\langle h_{1}, \ldots, h_{\mathrm{N}}|\overline{\mathrm{T}}(\lambda)| t\right\rangle=t(\lambda)\left\langle h_{1}, \ldots, h_{\mathrm{N}} \mid t\right\rangle,
$$

that is $t(\lambda) \in \Sigma_{\overline{\mathrm{T}}}$ and $|t\rangle$ is the corresponding $\overline{\mathrm{T}}$-eigenstate as $\overline{\mathrm{T}}(\lambda)$ is a polynomials of degree $\mathrm{N}-1$ in $\lambda$, even or odd for $\mathrm{N}$ odd or even. 
Note that the Baxter Q-operator construction given in [109] can be adapted in particular to the representation here considered and it represents an interesting issue as allows to reformulate by functional equations the SOV characterization of $\overline{\mathrm{T}}$-spectrum.

\section{$5 \bar{T}$-decomposition of the identity}

As previously introduced in [1, 2] and [3], we recall the definition of separate covector $\langle\alpha|$ and vector $|\beta\rangle$ in the SOV representations:

$$
\begin{aligned}
& \langle\alpha|=\sum_{h_{1}=0}^{2 s_{1}} \cdots \sum_{h_{N}=0}^{2 s_{\mathrm{N}}} \prod_{a=1}^{\mathrm{N}} \alpha_{a}\left(\eta_{a}^{\left(h_{a}\right)}\right) \prod_{1 \leq b<a \leq \mathrm{N}}\left(\eta_{a}^{\left(h_{a}\right)}-\eta_{b}^{\left(h_{b}\right)}\right)\left\langle h_{1}, \ldots, h_{\mathrm{N}}\right|, \\
& |\beta\rangle=\sum_{h_{1}=0}^{2 s_{1}} \cdots \sum_{h_{\mathrm{N}}=0}^{2 s_{\mathrm{N}}} \prod_{a=1}^{\mathrm{N}} \beta_{a}\left(\eta_{a}^{\left(h_{a}\right)}\right) \prod_{1 \leq b<a \leq \mathrm{N}}\left(\eta_{a}^{\left(h_{a}\right)}-\eta_{b}^{\left(h_{b}\right)}\right)\left|h_{1}, \ldots, h_{\mathrm{N}}\right\rangle,
\end{aligned}
$$

and we show that also for the model under consideration the following results hold:

Proposition 5.1. The generic separate covector $\langle\alpha|$ acts on the generic separate vector $|\beta\rangle$ according to the following formula:

$$
\langle\alpha \mid \beta\rangle=\operatorname{det}_{\mathrm{N}}\left\|\mathcal{M}_{a, b}^{(\alpha, \beta)}\right\| \text { with } \mathcal{M}_{a, b}^{(\alpha, \beta)} \equiv \sum_{h=0}^{2 s_{a}} \alpha_{a}\left(\eta_{a}^{(h)}\right) \beta_{a}\left(\eta_{a}^{(h)}\right)\left(\eta_{a}^{(h)}\right)^{b-1} .
$$

Proof. The proof is a consequence of formula (3.19) which implies:

$$
\langle\alpha \mid \beta\rangle=\sum_{h_{1}=0}^{2 s_{1}} \ldots \sum_{h_{\mathrm{N}}=0}^{2 s_{\mathrm{N}}} V\left(\eta_{1}^{\left(h_{1}\right)}, \ldots, \eta_{\mathrm{N}}^{\left(h_{\mathrm{N}}\right)}\right) \prod_{a=1}^{\mathrm{N}} \alpha_{a}\left(\eta_{a}^{\left(h_{a}\right)}\right) \beta_{a}\left(\eta_{a}^{\left(h_{a}\right)}\right),
$$

where $V\left(x_{1}, \ldots, x_{\mathrm{N}}\right) \equiv \prod_{1 \leq b<a \leq \mathrm{N}}\left(x_{a}-x_{b}\right)$ is the Vandermonde determinant, and of the multilinearity of the determinant.

The scalar product introduced in Section 2.1.1 allows to characterize $\langle\alpha|$ as the dual of a vector $(\langle\alpha|)^{\dagger} \in \mathcal{R}_{\mathrm{N}}$, then the previous formula represents also the scalar product of two states in $\mathcal{R}_{\mathrm{N}}$. Indeed, from the Hermitian conjugation properties (2.23) and (2.25), it follows that $(\langle\alpha|)^{\dagger}$ is a separate vector w.r.t. the A-decomposition of the identity. Finally, let us remark that for the transfer matrix the orthogonality of eigenstates corresponding to different eigenvalues can be proven directly by using the previous scalar product formula:

Corollary 5.1. Let us take $t(\lambda)$ and $t^{\prime}(\lambda) \in \Sigma_{\overline{\mathbf{T}}}$ and let $\langle t|$ and $\left|t^{\prime}\right\rangle$ be the corresponding $\bar{\top}_{\text {-eigenstates characterized in }}$ Theorem 4.1 then for $t(\lambda) \neq t^{\prime}(\lambda)$ the $\mathrm{N} \times \mathrm{N}$ matrix $\left\|\mathcal{M}_{a, b}^{\left(t, t^{\prime}\right)}\right\|$ has rank equal or smaller than $\mathrm{N}-1$. Indeed, the non-zero $\mathrm{N} \times 1$ vector $V^{\left(t, t^{\prime}\right)}$ of components:

$$
V_{b}^{\left(t, t^{\prime}\right)} \equiv c_{b}^{\prime}-c_{b} \quad \forall b \in\{1, \ldots, \mathbf{N}\}
$$

where:

$$
t(\lambda)=\sum_{b=1}^{\mathrm{N}} c_{b} \lambda^{b-1}, \quad t^{\prime}(\lambda)=\sum_{b=1}^{\mathrm{N}} c_{b}^{\prime} \lambda^{b-1}
$$

is an eigenvector of $\left\|\mathcal{M}_{a, b}^{\left(t, t^{\prime}\right)}\right\|$ corresponding to the eigenvalue zero.

Proof. By the definition (5.5), (5.6) and the definition of $\mathcal{M}_{a, b}^{\left(t, t^{\prime}\right)}$ in (5.3), it holds:

$$
\sum_{b=1}^{\mathrm{N}} \mathcal{M}_{a, b}^{\left(t, t^{\prime}\right)} \mathrm{V}_{b}^{\left(t, t^{\prime}\right)}=\sum_{h=0}^{2 s_{a}} Q_{t^{\prime}}\left(\eta_{a}^{(h)}\right) \bar{Q}_{t}\left(\eta_{a}^{(h)}\right)\left(t^{\prime}\left(\eta_{a}^{(h)}\right)-t\left(\eta_{a}^{(h)}\right)\right),
$$


then by using the Baxter relations (4.5)-(4.6) and (4.8)-(4.9) we can write:

$$
\begin{aligned}
Q_{t^{\prime}}\left(\eta_{a}^{(h)}\right) \bar{Q}_{t}\left(\eta_{a}^{(h)}\right)\left(t^{\prime}\left(\eta_{a}^{(h)}\right)-t\left(\eta_{a}^{(h)}\right)\right) & =\left(d\left(\eta_{a}^{\left(h+1-\beta_{h}\right)}\right) Q_{t^{\prime}}\left(\eta_{a}^{(h+1)}\right)+a\left(\eta_{a}^{\left(h-1+\alpha_{h}\right)}\right) Q_{t^{\prime}}\left(\eta_{a}^{(h-1)}\right)\right) \bar{Q}_{t}\left(\eta_{a}^{(h)}\right) \\
& -\left(a\left(\eta_{a}^{(h)}\right) \bar{Q}_{t}\left(\eta_{a}^{(h+1)}\right)+d\left(\eta_{a}^{(h)}\right) \bar{Q}_{t}\left(\eta_{a}^{(h-1)}\right)\right) Q_{t^{\prime}}\left(\eta_{a}^{(h)}\right)
\end{aligned}
$$

and by substituting them in 5.7 we get our result:

$$
\sum_{b=1}^{\mathrm{N}} \mathcal{M}_{a, b}^{\left(t, t^{\prime}\right)} \mathrm{V}_{b}^{\left(t, t^{\prime}\right)}=0 \quad \forall a \in\{1, \ldots, \mathrm{N}\} .
$$

The normality of $\overline{\mathbf{T}}(\lambda)$ and the simplicity of its spectrum imply the following decomposition of the identity:

$$
\mathbb{I}=\sum_{t(\lambda) \in \Sigma_{\bar{\top}}} \frac{|t\rangle\langle t|}{\langle t \mid t\rangle} \text { with }\langle t \mid t\rangle=\operatorname{det}_{\mathrm{N}}\left\|\mathcal{M}_{a, b}^{(t, t)}\right\|,
$$

where for the $\overline{\mathrm{T}}$-eigenstates $\langle t|$ and $|t\rangle$ we are using the characterization given in Theorem 4.1 .

\section{Reconstruction of local spin generators}

\subsection{Antiperiodic solution of the quantum inverse problem}

Here we show how to write the solution of the quantum inverse problem in the case of the antiperiodic XXX spin $\left\{s_{1}, \ldots, s_{\mathrm{N}}\right\}$ chain. The solution here presented is a simple consequence of the results derived in [45, 4] for the periodic chain.

Proposition 6.1. In the $X X X \operatorname{spin}\left\{s_{1}, \ldots, s_{\mathrm{N}}\right\}$-chain, the generic local operator $X_{n} \in \operatorname{End}\left(V_{n}^{\left(s_{n}\right)}\right)$ at any quantum site $n \in$ $\{1, \ldots, N\}$ admits the following reconstruction:

$$
X_{n}=\prod_{k=1}^{n-1} \overline{\mathbf{T}}^{\left(s_{k}\right)}\left(\eta_{k}\right) t r_{0_{n}}\left(X_{0_{n}} \overline{\mathrm{M}}_{0_{n}}^{\left(s_{n}\right)}\left(\eta_{n}\right)\right) \prod_{k=1}^{n}\left(\overline{\mathrm{T}}^{\left(s_{k}\right)}\left(\eta_{k}\right)\right)^{-1}
$$

where the auxiliary space $0_{n}$ is isomorphic to $R_{n}^{\left(s_{n}\right)}$. Moreover, for $X_{n} \equiv S_{n}^{\alpha}$ and $\alpha \equiv \pm, z$, the following decompositions hold:

$$
\begin{aligned}
\operatorname{tr}_{0_{n}}\left(S_{0_{n}}^{\alpha} \overline{\mathrm{M}}_{0_{n}}^{\left(s_{n}\right)}\left(\eta_{n}\right)\right) & =\sum_{k=1}^{2 s_{n}} \overline{\mathbf{T}^{\left(s_{n}-\frac{k}{2}\right)}}\left(\eta_{n}+\frac{k \eta}{2}\right) \operatorname{tr}_{0}\left(S_{0}^{\alpha} \overline{\mathrm{M}}_{0}^{(1 / 2)}\left(\eta_{n}^{-}+\left(k-s_{n}\right) \eta\right)\right) \\
& \times \overline{\mathbf{T}}^{\left(\frac{k-1}{2}\right)}\left(\eta_{n}^{-}+\frac{\left(k-2 s_{n}\right) \eta}{2}\right), \\
& =\sum_{k=1}^{2 s_{n}} \overline{\mathbf{T}}\left(\frac{k-1}{2}\right)\left(\eta_{n}^{-}+\frac{\left(k-2 s_{n}\right) \eta}{2}\right) \operatorname{tr}_{0}\left(S_{0}^{\alpha} \overline{\mathrm{M}}_{0}^{(1 / 2)}\left(\eta_{n}^{-}+\left(k-s_{n}\right) \eta\right)\right) \\
& \times \overline{\mathbf{T}}^{\left(s_{n}-\frac{k}{2}\right)}\left(\eta_{n}+\frac{k \eta}{2}\right)
\end{aligned}
$$

in terms of the fused transfer matrix and the matrix elements of the basic $\overline{\mathrm{M}}_{0}^{(1 / 2)}(\lambda)$ monodromy matrix.

Proof. From Proposition 1 of the article [45], it holds:

$$
X_{n}=\prod_{k=1}^{n-1} \mathrm{~T}^{\left(s_{k}\right)}\left(\eta_{k}\right) t r_{0_{n}}\left(X_{0_{n}} \mathrm{M}_{0_{n}}^{\left(s_{n}\right)}\left(\eta_{n}\right)\right) \prod_{k=1}^{n}\left(\mathrm{~T}^{\left(s_{k}\right)}\left(\eta_{k}\right)\right)^{-1}
$$


then it holds:

$$
\Sigma_{n}^{(x)}=\prod_{k=1}^{n-1} \mathrm{~T}^{\left(s_{k}\right)}\left(\eta_{k}\right) \overline{\mathbf{T}}^{\left(s_{n}\right)}\left(\eta_{n}\right) \prod_{k=1}^{n}\left(\mathrm{~T}^{\left(s_{k}\right)}\left(\eta_{k}\right)\right)^{-1}
$$

So, we can use (6.5) to write:

$$
\begin{aligned}
\prod_{b=1}^{c} \Sigma_{b}^{(x)} & =\prod_{b=1}^{c} \overline{\mathbf{T}}^{\left(s_{b}\right)}\left(\eta_{b}\right) \prod_{b=1}^{c}\left(\mathbf{T}^{\left(s_{b}\right)}\left(\eta_{b}\right)\right)^{-1} \\
& =\prod_{b=1}^{c} \mathbf{T}^{\left(s_{b}\right)}\left(\eta_{b}\right) \prod_{b=1}^{c}\left(\overline{\mathbf{T}}^{\left(s_{b}\right)}\left(\eta_{b}\right)\right)^{-1}
\end{aligned}
$$

where the second equality follows from:

$$
\prod_{b=1}^{c} \Sigma_{b}^{(x)}=\prod_{b=1}^{c}\left(\Sigma_{b}^{(x)}\right)^{-1}
$$

being:

$$
\Sigma_{b}^{(x)} \Sigma_{b}^{(x)}=\mathbb{I}_{b} \quad \text { where } \mathbb{I}_{b} \text { is the identity matrix in } \mathrm{R}_{b}^{\left(s_{b}\right)} .
$$

The result (6.1) is derived computing:

$$
X_{n}=\prod_{b=1}^{n-1} \Sigma_{b}^{(x)} \tilde{X}_{n} \prod_{b=1}^{n} \Sigma_{b}^{(x)} \quad \text { with } \quad \tilde{X}_{n}=X_{n} \Sigma_{n}^{(x)}
$$

and using the reconstruction (6.4) for $\tilde{X}_{n}$ and the reconstruction in (6.6) for the first product of $\Sigma_{b}^{(x)}$ while the reconstruction in (6.7) for the second product of $\Sigma_{b}^{(x)}$. The formula (6.2) can be proven following step by step the proof given in [45] only changing the periodic objects with the antiperiodic ones. In the same way we can prove formula (6.3) following step by step the proof given in [4].

\section{Form factors of local operators}

Proposition 7.1. Let $\langle t|$ and $\left|t^{\prime}\right\rangle$ be two eigenstates of the transfer matrix $\overline{\mathrm{T}}(\lambda)$, then it holds:

$$
\left\langle t\left|S_{n}^{-}\right| t^{\prime}\right\rangle=\frac{\prod_{h=1}^{n-1} t^{\left(s_{h}\right)}\left(\eta_{h}\right)}{\prod_{h=1}^{n} t^{\prime\left(s_{n}\right)}\left(\eta_{h}\right)} \operatorname{det}_{\mathrm{N}+1}\left(\left\|\mathcal{S}_{a, b}^{\left(-, t, t^{\prime}\right)}\right\|\right)
$$

where $\left\|\mathcal{S}_{a, b}^{\left(-, t, t^{\prime}\right)}\right\|$ is the $(\mathrm{N}+1) \times(\mathrm{N}+1)$ matrix:

$$
\begin{aligned}
\mathcal{S}_{a, b}^{\left(-, t, t^{\prime}\right)} & \equiv \mathcal{M}_{a, b}^{\left(t, t^{\prime}\right)} \text { for } a \in\{1, \ldots, \mathrm{N}\}, b \in\{1, \ldots, \mathrm{N}+1\} \\
\mathcal{S}_{\mathrm{N}+1, b}^{\left(-, t, t^{\prime}\right)} & \equiv \sum_{k=1}^{2 s_{n}} \frac{t^{\left(s_{n}-\frac{k}{2}\right)}\left(\bar{\eta}_{n}^{\left(s_{n}+(k+1) / 2\right)}\right)}{t^{\prime\left(\frac{k-1}{2}\right)}\left(\bar{\eta}_{n}^{(k / 2)}\right)}\left(\bar{\eta}_{n}^{(k)}\right)^{b-1}, \quad b \in\{1, \ldots, \mathrm{N}+1\},
\end{aligned}
$$

where we have used the notation:

$$
\bar{\eta}_{n}^{\left(k_{n}\right)} \equiv \eta_{n}+\left(k_{n}-\left(s_{n}+1 / 2\right)\right) \eta
$$

Proof. We can compute the action of $S_{n}^{-}$, by using the reconstruction:

$$
S_{n}^{-}=\prod_{k=1}^{n-1} \overline{\mathbf{T}}^{\left(s_{k}\right)}\left(\eta_{k}\right) \sum_{k=1}^{2 s_{n}} \overline{\mathbf{T}}^{\left(s_{n}-\frac{k}{2}\right)}\left(\bar{\eta}_{n}^{\left(s_{n}+(k+1) / 2\right)}\right) D\left(\bar{\eta}_{n}^{(k)}\right) \overline{\mathbf{T}}^{\left(\frac{k-1}{2}\right)}\left(\bar{\eta}_{n}^{(k / 2)}\right) \prod_{k=1}^{n}\left(\overline{\mathbf{T}}^{\left(s_{k}\right)}\left(\eta_{k}\right)\right)^{-1}
$$


so it holds:

$$
\left\langle t\left|S_{n}^{-}\right| t^{\prime}\right\rangle=\frac{\prod_{h=1}^{n-1} t^{\left(s_{h}\right)}\left(\eta_{h}\right)}{\prod_{h=1}^{n} t^{\prime\left(s_{n}\right)}\left(\eta_{h}\right)} \sum_{k=1}^{2 s_{n}} \frac{t^{\left(s_{n}-\frac{k}{2}\right)}\left(\bar{\eta}_{n}^{\left(s_{n}+(k+1) / 2\right)}\right)}{t^{\prime\left(\frac{k-1}{2}\right)}\left(\bar{\eta}_{n}^{(k / 2)}\right)}\left\langle t\left|D\left(\bar{\eta}_{n}^{(k)}\right)\right| t^{\prime}\right\rangle
$$

Now from the right SOV representation, we have:

$$
D\left(\bar{\eta}_{n}^{(k)}\right)\left|t^{\prime}\right\rangle=\sum_{h_{1}=0}^{2 s_{1}} \cdots \sum_{h_{\mathrm{N}}=0}^{2 s_{\mathrm{N}}} \prod_{a=1}^{\mathrm{N}}\left(\bar{\eta}_{n}^{(k)}-\eta_{a}^{\left(h_{a}\right)}\right) Q_{t^{\prime}}\left(\eta_{a}^{\left(h_{a}\right)}\right) \prod_{1 \leq b<a \leq \mathrm{N}}\left(\eta_{a}^{\left(h_{a}\right)}-\eta_{b}^{\left(h_{b}\right)}\right)\left|h_{1}, \ldots, h_{\mathrm{N}}\right\rangle,
$$

and we can rewrite the coefficient as:

$$
\prod_{a=1}^{\mathrm{N}} Q_{t^{\prime}}\left(\eta_{a}^{\left(h_{a}\right)}\right) V\left(\eta_{1}^{\left(h_{1}\right)}, \ldots, \eta_{\mathrm{N}}^{\left(h_{\mathrm{N}}\right)}, \bar{\eta}_{n}^{(k)}\right)
$$

where $V()$ is the determinant of the $(\mathrm{N}+1) \times(\mathrm{N}+1)$ Vandermonde matrix $\left\|V_{i, j}\right\|$ defined by:

$$
V_{i, j} \equiv\left(\eta_{i}^{\left(h_{i}\right)}\right)^{j-1} \quad \forall i \in\{1, \ldots, \mathrm{N}\}, V_{\mathrm{N}+1, j} \equiv\left(\bar{\eta}_{n}^{(k)}\right)^{j-1} \forall j \in\{1, \ldots, \mathrm{N}+1\} .
$$

Now taking the scalar product and resumming we get the result.

Proposition 7.2. Let $\langle t|$ and $\left|t^{\prime}\right\rangle$ be two eigenstates of the transfer matrix $\overline{\mathrm{T}}(\lambda)$, then it holds:

$$
\left\langle t\left|S_{n}^{z}\right| t^{\prime}\right\rangle=\operatorname{det}_{\mathrm{N}+1}\left(\left\|\mathcal{S}_{a, b}^{\left(z, t, t^{\prime}\right)}\right\|\right)
$$

where $\left\|\mathcal{S}_{a, b}^{\left(z, t, t^{\prime}\right)}\right\|$ is the $(\mathrm{N}+1) \times(\mathrm{N}+1)$ matrix:

$$
\begin{aligned}
\mathcal{S}_{a, b}^{\left(z, t, t^{\prime}\right)} & \equiv \mathcal{M}_{a, b}^{\left(t, t^{\prime}\right)} \quad \text { for } a \in\{1, \ldots, \mathrm{N}\}, \quad b \in\{1, \ldots, \mathrm{N}\} \\
\mathcal{S}_{\mathrm{N}+1, b}^{\left(z, t, t^{\prime}\right)} & \equiv \frac{\prod_{h=1}^{n-1} t^{\left(s_{h}\right)}\left(\eta_{h}\right)}{\prod_{h=1}^{n} t^{\prime\left(s_{n}\right)}\left(\eta_{h}\right)} \sum_{h=0}^{2 s_{n}-1} \frac{t^{(h / 2)}\left(\eta_{n}^{((h+1) / 2)}\right)}{t^{\prime\left(s_{n}-\frac{(h+1)}{2}\right)}\left(\eta_{n}^{\left(h / 2+s_{n}\right)}\right)}\left(\eta_{n}^{(h)}\right)^{b-1} \quad \text { for } b \in\{1, \ldots, \mathrm{N}\} \\
\mathcal{S}_{a, \mathrm{~N}+1}^{\left(z, t, t^{\prime}\right)} & \equiv \sum_{h_{a}=0}^{2 s_{a}} Q_{t^{\prime}}\left(\eta_{a}^{\left(h_{a}\right)}\right) \bar{Q}_{t}\left(\eta_{a}^{\left(h_{a}-1\right)}\right) d\left(\eta_{a}^{\left(h_{a}\right)}\right) \text { for } a \in\{1, \ldots, \mathrm{N}\}, \\
\mathcal{S}_{\mathrm{N}+1, \mathrm{~N}+1}^{\left(z, t t^{\prime}\right)} & \equiv-s_{n} .
\end{aligned}
$$

Proof. We can compute the action of $S_{n}^{z}$, by using the reconstruction:

$$
S_{n}^{z}=\prod_{k=1}^{n-1} \overline{\mathbf{T}}^{\left(s_{k}\right)}\left(\eta_{k}\right) \sum_{h=0}^{2 s_{n}-1} \overline{\mathbf{T}}^{(h / 2)}\left(\eta_{n}^{\left(\frac{h+1}{2}\right)}\right)\left(\frac{C\left(\eta_{n}^{(h)}\right)-B\left(\eta_{n}^{(h)}\right)}{2}\right) \overline{\mathbf{T}}^{\left(s_{n}-\frac{(h+1)}{2}\right)}\left(\eta_{n}^{\left(h / 2+s_{n}\right)}\right) \prod_{k=1}^{n}\left(\overline{\mathbf{T}}^{\left(s_{k}\right)}\left(\eta_{k}\right)\right)^{-1}
$$

so it holds:

$$
\left\langle t\left|S_{n}^{z}\right| t^{\prime}\right\rangle=\frac{\prod_{h=1}^{n-1} t^{\left(s_{h}\right)}\left(\eta_{h}\right)}{\prod_{h=1}^{n} t^{\prime\left(s_{n}\right)}\left(\eta_{h}\right)} \sum_{h=0}^{2 s_{n}-1} \frac{t^{(h / 2)}\left(\eta_{n}^{((h+1) / 2)}\right)}{t^{\prime\left(s_{n}-\frac{(h+1)}{2}\right)}\left(\eta_{n}^{\left(h / 2+s_{n}\right)}\right)}\left\langle t\left|C\left(\eta_{n}^{(h)}\right)\right| t^{\prime}\right\rangle-s_{n}\left\langle t \mid t^{\prime}\right\rangle .
$$

Here we have used the property:

$$
\sum_{h=0}^{2 s_{n}-1} \overline{\mathbf{T}}^{(h)}\left(\eta_{n}^{\left(\frac{h+1}{2}\right)}\right) \overline{\mathbf{T}}\left(\eta_{n}^{(h)}\right) \overline{\mathbf{T}}^{\left(s_{n}-\frac{(h+1)}{2}\right)}\left(\eta_{n}^{\left(h / 2+s_{n}\right)}\right)=2 s_{n} \overline{\mathbf{T}}^{\left(s_{n}\right)}\left(\eta_{n}\right),
$$

which is proven showing that it holds for the eigenvalues following step by step the proof given for the formula (A.3) in [4]. Now from the right SOV representation of $C\left(\eta_{n}\right)$, we have:

$$
\begin{aligned}
C\left(\eta_{n}^{(h)}\right)\left|t^{\prime}\right\rangle= & \sum_{a=1}^{\mathrm{N}} \sum_{h_{1}=0}^{2 s_{1}} \cdots \sum_{h_{\mathrm{N}}=0}^{2 s_{\mathrm{N}}} \prod_{b=1}^{\mathrm{N}} Q_{t^{\prime}}\left(\eta_{b}^{\left(h_{b}\right)}\right) \prod_{b \neq a, b=1}^{\mathrm{N}}\left[\frac{\eta_{n}^{(h)}-\eta_{b}^{\left(h_{b}\right)}}{\eta_{a}^{\left(h_{a}\right)}-\eta_{b}^{\left(h_{b}\right)}} d\left(\eta_{a}^{\left(h_{a}\right)}\right)\right] \\
& \times \prod_{1 \leq b<a \leq \mathrm{N}}\left(\eta_{a}^{\left(h_{a}\right)}-\eta_{b}^{\left(h_{b}\right)}\right)\left|h_{1}, \ldots, h_{a}-1, \ldots, h_{\mathrm{N}}\right\rangle,
\end{aligned}
$$


and so we can write:

$$
\begin{aligned}
\left\langle t\left|C\left(\eta_{n}^{(h)}\right)\right| t^{\prime}\right\rangle= & \sum_{a=1}^{\mathrm{N}}(-1)^{\mathrm{N}+1+a} \sum_{h_{1}=0}^{2 s_{1}} \cdots \sum_{h_{\mathrm{N}}=0}^{2 s_{\mathrm{N}}} \widehat{V}_{a}\left(\eta_{1}^{\left(h_{1}\right)}, \ldots, \eta_{\mathrm{N}}^{\left(h_{\mathrm{N}}\right)}, \eta_{n}^{(h)}\right) \\
& \times Q_{t^{\prime}}\left(\eta_{a}^{\left(h_{a}\right)}\right) \bar{Q}_{t}\left(\eta_{a}^{\left(h_{a}-1\right)}\right) d\left(\eta_{a}^{\left(h_{a}\right)}\right) \prod_{b \neq a, b=1}^{\mathrm{N}} Q_{t^{\prime}}\left(\eta_{b}^{\left(h_{b}\right)}\right) \bar{Q}_{t}\left(\eta_{b}^{\left(h_{b}\right)}\right),
\end{aligned}
$$

where $(-1)^{\mathrm{N}+1+a} \widehat{V}_{a}\left(\eta_{1}^{\left(h_{1}\right)}, \ldots ., \eta_{\mathrm{N}}^{\left(h_{\mathrm{N}}\right)}, \eta_{n}^{(h)}\right)$ is the cofactor $(a, \mathrm{~N}+1)$ of the $(\mathrm{N}+1) \times(\mathrm{N}+1)$ Vandermonde matrix:

$$
V_{i, j} \equiv\left(\eta_{i}^{\left(h_{i}\right)}\right)^{j-1} \quad \forall i \in\{1, \ldots, \mathrm{N}\}, V_{\mathrm{N}+1, j} \equiv\left(\eta_{n}^{(h)}\right)^{j-1} \forall j \in\{1, \ldots, \mathrm{N}+1\} .
$$

It is trivial to remark that the above sum is the develop of the determinant of a $(\mathrm{N}+1) \times(\mathrm{N}+1)$ matrix and then:

$$
\frac{\prod_{h=1}^{n-1} t^{\left(s_{h}\right)}\left(\eta_{h}\right)}{\prod_{h=1}^{n} t^{\prime\left(s_{n}\right)}\left(\eta_{h}\right)} \sum_{h=0}^{2 s_{n}-1} \frac{t^{(h / 2)}\left(\eta_{n}^{((h+1) / 2)}\right)}{t^{\prime\left(s_{n}-\frac{(h+1)}{2}\right)}\left(\eta_{n}^{\left(h / 2+s_{n}\right)}\right)}\left\langle t\left|C\left(\eta_{n}^{(h)}\right)\right| t^{\prime}\right\rangle=\operatorname{det}_{\mathrm{N}+1}\left\|\mathrm{~s}_{a, b}^{\left(t, t^{\prime}\right)}\right\|
$$

with:

$$
\begin{aligned}
& \mathrm{S}_{a, b}^{\left(t, t^{\prime}\right)} \equiv \mathcal{M}_{a, b}^{\left(t, t^{\prime}\right)} \quad \text { for } a \in\{1, \ldots, \mathrm{N}\}, \quad b \in\{1, \ldots, \mathrm{N}\} \\
& \mathrm{S}_{\mathrm{N}+1, b}^{\left(t, t^{\prime}\right)} \equiv \frac{\prod_{h=1}^{n-1} t^{\left(s_{h}\right)}\left(\eta_{h}\right)}{\prod_{h=1}^{n} t^{\prime\left(s_{n}\right)}\left(\eta_{h}\right)} \sum_{h=0}^{2 s_{n}-1} \frac{t^{(h / 2)}\left(\eta_{n}^{((h+1) / 2)}\right)}{t^{\prime\left(s_{n}-\frac{(h+1)}{2}\right)}\left(\eta_{n}^{\left(h / 2+s_{n}\right)}\right)}\left(\eta_{n}^{(h)}\right)^{b-1} \quad \text { for } b \in\{1, \ldots, \mathrm{N}\} \\
& \mathrm{S}_{a, \mathrm{~N}+1}^{\left(t, t^{\prime}\right)} \equiv \sum_{h_{a}=0}^{2 s_{a}} Q_{t^{\prime}}\left(\eta_{a}^{\left(h_{a}\right)}\right) \bar{Q}_{t}\left(\eta_{a}^{\left(h_{a}-1\right)}\right) d\left(\eta_{a}^{\left(h_{a}\right)}\right) \text { for } a \in\{1, \ldots, \mathrm{N}\} \text {, } \\
& \mathrm{S}_{\mathrm{N}+1, \mathrm{~N}+1}^{\left(t, t^{\prime}\right)} \equiv 0 \text {. }
\end{aligned}
$$

Then if we rewrite:

$$
\left\langle t \mid t^{\prime}\right\rangle=\underset{\mathrm{N}+1}{\operatorname{det}}\left\|\mathrm{N}_{a, b}^{\left(t, t^{\prime}\right)}\right\|
$$

where we have defined the matrix:

$$
\begin{aligned}
\mathrm{N}_{a, b}^{\left(t, t^{\prime}\right)} & \equiv \mathcal{M}_{a, b}^{\left(t, t^{\prime}\right)} \quad \text { for } a \in\{1, \ldots, \mathrm{N}\}, \quad b \in\{1, \ldots, \mathrm{N}\} \\
\mathrm{N}_{\mathrm{N}+1, b}^{\left(t, t^{\prime}\right)} & \equiv \frac{\prod_{h=1}^{n-1} t^{\left(s_{h}\right)}\left(\eta_{h}\right)}{\prod_{h=1}^{n} t^{\left(s_{n}\right)}\left(\eta_{h}\right)} \sum_{h=0}^{2 s_{n}-1} \frac{t^{(h / 2)}\left(\eta_{n}^{((h+1) / 2)}\right)}{t^{\prime\left(s_{n}-\frac{(h+1)}{2}\right)}\left(\eta_{n}^{\left(h / 2+s_{n}\right)}\right)}\left(\eta_{n}^{(k)}\right)^{b-1} \quad \text { for } b \in\{1, \ldots, \mathrm{N}\} \\
\mathrm{N}_{a, \mathrm{~N}+1}^{\left(t, t^{\prime}\right)} & \equiv 0 \text { for } a \in\{1, \ldots, \mathrm{N}\} \\
\mathrm{N}_{\mathrm{N}+1, \mathrm{~N}+1}^{\left(t, t^{\prime}\right)} & \equiv 1 .
\end{aligned}
$$

we finally get our determinant formula.

\section{Conclusion and outlook}

For the higher spin representations of the rational 6-vertex Yang-Baxter algebra we have completely characterized the transfer matrix spectrum by separation of variables and proven its simplicity. Moreover, we have provided the SOV-reconstruction of all local operators and determinant formulae for the scalar product of separate states. Finally, we were able to compute the form factors of the local spin operators getting one determinant formulae similar to those of the scalar products. 
The relevance of these findings is clear as it represents the first fundamental step to characterize the dynamics of this quantum model. Indeed, the decomposition of the identity (5.10) allows to expand any m-point function:

$$
\frac{\left\langle t\left|X_{r_{1}} \cdots X_{r_{\mathrm{g}+1}}\right| t\right\rangle}{\langle t \mid t\rangle}=\sum_{t_{1}(\lambda), \ldots, t_{\mathrm{g}}(\lambda) \in \Sigma_{\overline{\mathrm{T}}}} \frac{\left\langle t\left|X_{r_{1}}\right| t_{1}\right\rangle\left\langle t_{\mathrm{g}}\left|X_{r_{\mathrm{g}}}\right| t\right\rangle \prod_{j=2}^{\mathrm{g}}\left\langle t_{j-1}\left|X_{r_{j}}\right| t_{j}\right\rangle}{\langle t \mid t\rangle \prod_{j=1}^{\mathrm{g}}\left\langle t_{j} \mid t_{j}\right\rangle} \text { with } X_{n} \in \operatorname{End}\left(R_{n}^{\left(s_{n}\right)}\right),
$$

in terms of the form factors $\left\langle t\left|O_{n}\right| t^{\prime}\right\rangle$. Then m-point functions can be potentially analyzed by extending and adapting to the current model the tools that were developed 13 in $[122]-[128]$ for the spin $1 / 2$ XXZ quantum chains in the ABA framework.

We would like to underline that the results presented in this paper provide a further confirmation of the universality in the characterization of form factors of local operators of integrable quantum models when described by our approach in SOV framework. In particular, the comparison of the current results with those obtained previously by the same approach in [1], for the cyclic representations of the trigonometric 6-vertex Yang-Baxter algebra, in [2], for the spin-1/2 h.w. representations of the same algebra, and in [3], for the spin 1/2 representations of the 6-vertex trigonometric reflection algebra, makes clear this universal picture. Indeed, a part from model dependent features, like the nature of the spectrum of the quantum separate variables and the SOV-reconstruction of local operators, the form factors admit always the same type of determinant representations whose matrix elements are "convolutions" over the spectrum of the separate variables of Baxter equations solutions plus contributions coming from the local operators. The same type of statements will be proven for the SOS model with antiperiodic boundary conditions and for the XYZ spin-1/2 quantum chain with periodic boundary conditions. In particular, in [106] the SOV characterization of the spectrum and the determinant formulae for the scalar products of separate states will be derived allowing in [129] to get determinant formulae for the form factors of local operators. It is worth recalling that in the literature there exist previous results on matrix elements of local operators which can be related to separation of variable methods. Of special interest is the Smirnov's paper [130] for the quantum integrable Toda chain [37] where the form factors of a conjectured 14 basis of local operators are derived in the Sklyanin's SOV framework by determinant formulae which respect the universal picture above outlined.

Finally, let us anticipate that the extension of the results derived in this paper to the antiperiodic XXZ spin $s$ quantum chains is currently under analysis. Note that while the SOV characterization of the spectrum and the scalar product formulae can be re-derived trivially along the same line described in this paper the main technical point to address remains the solution of the inverse problem for the local spin generators in a similar form to that presented in Section 6 Indeed, by using these type of reconstruction the form factors of the local spin generators will be derived following mainly the same steps presented in Section 7 The relevance of this project is evidenced by remarking that the XXZ spin- $s$ quantum chain is strictly related to the lattice discretization of the sine-Gordon model which is a fundamental prototype of integrable quantum field theory and then the solution of the XXZ spin-s quantum chain can provide a further way to solve the sine-Gordon quantum field theory according to the microscopic approach to integrable quantum field theories (IQFTs) described in the introduction of [1]. Our interest toward integrable microscopic approach to IQFTs is due to need to define an exact framework where to use the SOV reconstruction of local fields to overcome the longstanding problem of their identification ${ }^{15}$ in the S-matrix formulation 16 .

Acknowledgments I gratefully acknowledge the YITP Institute of Stony Brook for the opportunity to develop my research programs under support of National Science Foundation grants PHY-0969739. I would like to thank N. Grosjean, N. Kitanine, K. K. Kozlowski, J.-M. Maillet, B. M. McCoy and V. Terras for their interest in this paper. I would like also to thank the Theoretical Physics Group of the Laboratory of Physics at ENS-Lyon and the Mathematical Physics Group at IMB of the Dijon University for their hospitality (under support ANR-10-BLAN-0120-04-DIADEMS). Finally, I would like to thank H. Frahm for pointing out me some important references on the spectrum of higher spin XXX quantum chains and more general related quantum models.

\footnotetext{
${ }^{13}$ Note that physical observable as the dynamical structure factors $[115-121$ were accessible by this numerical approach.

${ }^{14}$ Note that in Smirnov's paper is the lack of a direct SOV reconstruction of local operators which forces the use of a conjecture on the basis of local operators. A reconstruction has been later presented in [131] not in the original Sklyanin's quantum separate variables but it was defined by a change of variables leading to a new set of quantum separate variables.

${ }^{15}$ Let us point out that many results are known which confirm the characterization of massive IQFTs as superrenormalizable perturbations by relevant local fields [132]-[135] of conformal field theories [136]-[140]; see for example [141]-[145] and the series of works [146]-[149], where the local field content of massive theories has been classified by that of the associated ultraviolet CFTs. However, these interesting results on the global operator structure of massive IQFTs do not solve completely the problem of the identification of specific local fields in the S-matrix formulation.

${ }^{16}$ See [150]-[153] and [154] for a review and references therein.
} 


\section{References}

[1] N. Grosjean, J. M. Maillet, G. Niccoli, On the form factors of local operators in the lattice sine-Gordon model, arXiv:1204.6307.

[2] G. Niccoli, Antiperiodic spin-1/2 XXZ quantum chains by separation of variables: Form factors and complete spectrum, arXiv:1205.4537.

[3] G. Niccoli, Non-diagonal open spin-1/2 XXZ quantum chain by separation of variables: Complete spectrum and matrix elements of some quasi-local operators, arXiv:1206.0646

[4] O. A. Castro-Alvaredo, J. M. Maillet, J. Phys. A 40 (2007) 7451.

[5] E. K. Sklyanin and L. D. Faddeev, Sov. Phys. Dokl. 23 (1978) 902.

[6] E. K. Sklyanin and L. A. Takhtajan, L.D. Faddeev, Theor. Math. Phys. 40 (1980) 688.

[7] L. D. Faddeev and L. A. Takhtajan, Russ. Math. Surveys, 34 : 5 (1979) 11.

[8] E. K. Sklyanin, Dokl. Akad. Nauk SSSR 244 (1979) 1337; Soviet Physics Dokl. 24 (1979) 107.

[9] P. P. Kulish and E. K. Sklyanin, Phys. Lett. A 70 (1979) 461.

[10] L. D. Faddeev, Sov. Sci. Rev. Math. Cl (1980) 107.

[11] E. K. Sklyanin, J. Sov. Math. 19 (1982) 1546.

[12] L. D. Faddeev, Les Houches lectures of 1982, Elsevier Sci. Publ. 563 (1984).

[13] L. D. Faddeev, How Algebraic Bethe Ansatz, works for integrable model, hep-th/9605187 1.

[14] M. Jimbo, Adv. Series in Math. Phys. 10, Singapore: World Scientific, (1990).

[15] P. P. Kulish and E. K. Sklyanin, Lect. Notes Phys. 151 (1982) 61.

[16] B. S. Shastry, Lect. Notes Phys, 242 (1985).

[17] H. B. Thacker, Rev. Mod. Phys. 53 (1981) 253.

[18] A. G. Izergin and V. E. Korepin, Nucl. Phys. B 205 (1982) 401.

[19] A. B. Zamolodchikov and V. A. Fateev, Soy. J. Nucl. Phys. 32 (1980) 298.

[20] L. A. Takhtajan, Phys. Lett. A 87 (1982) 479.

[21] K. Sogo, Y. Akutsu and T. Abe, Prog. Theor. Phys. 70 (1983) 30.

[22] K. Sogo, Phys. Lett. A 104 (1984) 51.

[23] M. Jimbo, Lett. Math. Phys. 10 (1985) 63.

[24] H.M. Babujian, Nucl. Phys. B 215 (1983) 317.

[25] H. M. Babujian and A. M. Tsvelick, Nucl. Phys. B 265 (1985) 24.

[26] P. P. Kulish, N. Y. Reshetikhin and E. K. Sklyanin, Lett. Math. Phys. 5 (1981) 393.

[27] A. N. Kirillov and N. Y. Reshetikhin, J. Phys. A: Math Gen. 20 (1987) 1565.

[28] E. C. Alcaraz, M. N. Barber and M. T. Batchelor, Ann. Phys. (N.Y.) 182 (1988) 280.

[29] D. Baranowski and V. Rittenberg, J. Phys. A 23 (1990) 1029.

[30] A. Klümper, M. T. Batchelor and E. A. Pearce, J. Phys. A 24 (1991) 3111.

[31] N. Kitanine, J. Phys. A: Math. Gen. 34 (2001) 8151.

[32] C.S. Melo, G.A.P. Ribeiro and M.J. Martins, Nucl. Phys. B, 711, 565 (2005).

[33] W. Galleas and M.J. Martins, Phys. Lett. A, 335, 167 (2005).

[34] R. J. Baxter, Exactly Solved Models in Statistical Mechanics, Academic Press, New York U.S.A. (1982).

[35] G. A. P. Ribeiro, M. J. Martins, W. Galleas, Nuclear Physics B 675 (2003) 567.

[36] G.A.P. Ribeiro and M.J. Martins, Nucl. Phys. B, 705, 521 (2005).

[37] E. K. Sklyanin, Lect. Notes Phys. 226 (1985) 196; E. K. Sklyanin J. Sov. Math. 31 (1985) 3417.

[38] E. K. Sklyanin, Quantum inverse scattering method. Selected topics. In: Quantum groups and quantum integrable systems (World Scientific, 1992) 63-97, arXiv:hep-th/9211111v1.

[39] E. K. Sklyanin, Prog. Theor. Phys. Suppl. 118 (1995) 35.

[40] H. Frahm, A. Seel, T. Wirth, Nucl. Phys. B 802 (2008) 351.

[41] H. Frahm, J. H. Grelik A. Seel, T. Wirth, J. Phys. A 44 (2011) 015001.

[42] L. Amico, H. Frahm, A. Osterloh, and T. Wirth, Nucl. Phys. B 839 [FS] (2010) 604.

[43] L. Amico, H. Frahm, A. Osterloh and G. A. P. Ribeiro, Nucl. Phys. B 787 (2007) 283.

[44] N. Kitanine, J. M. Maillet, and V. Terras, Nucl. Phys. B 554 (1999) 647.

[45] J. M. Maillet and V. Terras, Nucl. Phys. B 575 (2000) 627.

[46] A. G. Izergin, N. Kitanine, J. M. Maillet, and V. Terras, Nucl. Phys. B 554 (1999) 679.

[47] N. Kitanine, J. M. Maillet, and V. Terras, Nucl. Phys. B 567 (2000) 554.

[48] N. Kitanine, J. M. Maillet, N. A. Slavnov, and V. Terras, Nucl. Phys. B 641 (2002) 487.

[49] N. Kitanine, J. M. Maillet, N. A. Slavnov, and V. Terras, Nucl. Phys. B 642 (2002) 433.

[50] N. Kitanine, J. M. Maillet, N. A. Slavnov, and V. Terras, J. Phys. A 35 (2002) L385.

[51] N. Kitanine, J. M. Maillet, N. A. Slavnov, and V. Terras, J. Phys. A 35 (2002) L753.

[52] N. Kitanine, J. M. Maillet, N. A. Slavnov, and V. Terras, Nucl. Phys. B 712 (2005) 600.

[53] N. Kitanine, J. M. Maillet, N. A. Slavnov, and V. Terras, Nucl. Phys. B 729 (2005) 558.

[54] N. Kitanine, J. M. Maillet, N. A. Slavnov, and V. Terras, J. Phys. A 38 (2005) 7441.

[55] N. Kitanine, J. M. Maillet, N. A. Slavnov, and V. Terras, J. Stat. Mech. (2005) L09002.

[56] N. Kitanine, J. M. Maillet, N. A. Slavnov, and V. Terras, On the algebraic Bethe Ansatz approach to the correlation functions of the XXZ spin-1/2 Heisenberg chain, In Recent Progress in Solvable lattice Models, RIMS Sciences Project Research 2004 on Method of Algebraic Analysis in Integrable Systems, RIMS, Kyoto, Kokyuroku, 1480, 14 (2006); hep-th/0505006

[57] N. Kitanine, K. Kozlowski, J. M. Maillet, N. A. Slavnov, V. Terras, J. Stat. Mech. (2007) P01022. 
[58] N. Kitanine, K.K. Kozlowski, J.M. Maillet, G. Niccoli, N.A. Slavnov, V. Terras , J. Stat. Mech. (2007) P10009.

[59] K. K. Kozlowski, J.Stat.Mech. (2008) P02006.

[60] N. Kitanine, K. Kozlowski, J. M. Maillet, G. Niccoli, N. A. Slavnov, V. Terras, J. Stat. Mech. (2008) P07010.

[61] G. Niccoli and J. Teschner, J. Stat. Mech. (2010) P09014.

[62] G. Niccoli, Nucl. Phys. B 835 (2010) 263.

[63] G. Niccoli, JHEP JHEP03(2011)123.

[64] N. Grosjean and G. Niccoli, The $\tau_{2}$-model and the chiral Potts model revisited: Completeness of Bethe equations originated from Sklyanin SOV, arXiv:1205.4614

[65] V. V. Bazhanov, Y. G. Stroganov, J. Stat. Phys. 59 (1990) 799.

[66] R. J. Baxter, V. V. Bazhanov and J. H. H. Perk, Int. J. Mod. Phys. B4 (1990) 803.

[67] R. J. Baxter, J. Stat. Phys. 57 (1989) 1.

[68] G. Albertini, B. M. McCoy and J. H. H. Perk, Adv. Study in Pure Math. 19 (1989) 1.

[69] G. Albertini, B. M. McCoy and J. H. H. Perk, Phys. Lett. A 135 (1989) 159.

[70] G. Albertini, B. M. McCoy and J. H. H. Perk, Phys. Lett. A 139 (1989) 204.

[71] R. J. Baxter, J. H. H. Perk and H. Au-Yang, Phys. Lett. A 128 (1988) 138; H. Au-Yang and J. H. H. Perk, Adv. Studies Pure Math. 19 (1989).

[72] R. J. Baxter, Phys. Lett. A 133 (1989) 185

[73] H. Au-Yang, B. M. McCoy, J. H. H. Perk, S. Tang, and M. Yan, Phys. Lett. A 123 (1987) 219.

[74] B. M. McCoy, J. H. H. Perk, S. Tang, and C. H. Sah, Phys. Lett. A 125 (1987) 9.

[75] H. Au-Yang, B. M. McCoy, J. H. H. Perk, and S. Tang, Algebraic Analysis, Vol. 1, M. Kashiwara and T. Kawai, eds (Academic Press, New York, 1988).

[76] V. O. Tarasov Phys. Lett. A 147 (1990) 487.

[77] N. Grosjean, J. M. Maillet, G. Niccoli, On the matrix elements of local operators in the $\tau_{2}$-model and the chiral Potts model, to appear.

[78] G. von Gehlen, N. Iorgov, S. Pakuliak and V. Shadura, J. Phys. A: Math. Gen. 39 (2006) 7257.

[79] G. von Gehlen, N. Iorgov, S. Pakuliak, V. Shadura and Yu Tykhyy, J. Phys. A: Math. Theor. 40 (2007) 14117.

[80] G. von Gehlen, N. Iorgov, S. Pakuliak, V. Shadura and Yu Tykhyy, J. Phys. A: Math. Theor. 41 (2008) 095003.

[81] G. von Gehlen, N. Iorgov, S. Pakuliak, V. Shadura, J. Phys. A: Math. Theor. 42 (2009) 304026.

[82] R. J. Baxter, J. Stat. Phys. 117 (2004) 1.

[83] M. T. Batchelor, R. J. Baxter, M. J. O'Rourke, C. M. Yung, J. Phys. A 28 (1995) 2759.

[84] S. Niekamp, T. Wirth, H. Frahm, J. Phys. A 42 (2009) 195008.

[85] W. Heisenberg, Z. Phys. 49 (1928) 619.

[86] H. Bethe, Z. Phys. 71 (1931) 205.

[87] L. Hulthen, Ark. Mat. Astron. Fys. 26 (1938) 1.

[88] R. Orbach, Phys. Rev. 112 (1958) 309.

[89] L. R. Walker, Phys. Rev. 116 (1959) 1089.

[90] C. N. Yang and C. P. Yang, Phys. Rev. 150 (1966) 321.

[91] C. N. Yang and C. P. Yang, Phys. Rev. 150 (1966) 327.

[92] M. Gaudin, La Fonction d'onde de Bethe Paris, Masson, 1983.

[93] E. H. Lieb and D. C. Mattis, Mathematical Physics in One Dimension, New-York Academic, 1966.

[94] M. Gaudin, Phys. Rev. A 4 (1971) 386.

[95] F. C. Alcaraz, M. N. Barbe, M. T. Batchelor, R. J. Baxter and G. R. W. Quispel, J. Phys. A: Math. Gen. 20 (1987) 6397.

[96] E. K. Sklyanin, J. Phys. A: Math. Gen. 21 (1988) 2375.

[97] I. V. Cherednik, Theor. Math. Phys. 61 (1984) 977.

[98] P. P. Kulish and S. K. Sklyanin, J. Phys. A: Math. Gen. 24 (1991) L435.

[99] L. Mezincescu and R. Nepomechie, Int. J. Mod. Phys. A 6 (1991) 5231

[100] P. P. Kulish and E. K. Sklyanin, J. Phys. A: Math. Gen. 25 (1992) 5963.

[101] S. Ghoshal and A. Zamolodchikov, Int. J. Mod. Phys. A 9 (1994) 3841.

[102] S. Ghoshal and A. Zamolodchikov, Int. J. Mod. Phys. A 9 (1994) 4353.

[103] F. C. Alcaraz, M. N. Barber, M. T. Batchelor, R. J. Baxter and G. R. W. Quispel, J. Phys. A 20 (1987) 6397.

[104] N. Y. Reshetikhin, Lett. Math. Phys. 7 (1983) 205.

[105] N. Y. Reshetikhin, Sov. Phys. JETP 57 (1983) 691.

[106] G. Niccoli, Antiperiodic dynamical 6-vertex and periodic 8-vertex models: Scalar products and complete spectrum by separation of variables, to appear.

[107] A. G. Izergin and V. E. Korepin, Doklady Akademii Nauk 259 (1981) 76.

[108] A. G. Izergin and V. E. Korepin, A lattice model related to the nonlinear Schroedinger equation, arXiv:0910.0295

[109] C. M. Yung, M. T. Batchelor, Nucl. Phys. B 446 (1995) 461.

[110] J. M. Maillet, and J. Sanchez de Santos, Amer. Math. Soc. Transl. 201 (2000) 137.

[111] V. G. Drinfel'd, Soviet Math. Dokl. 28 (1983) 667.

[112] V. G. Drinfel'd, Proceedings of the the International Congress of Mathematicians, Berkeley, USA, 1986, 798-820, AMS, 1987.

[113] V. G. Drinfel'd, Leningrad Math. J. 1 (1990) 1419.

[114] V. Terras, Lett. Math. Phys. 48 (1999) 263.

[115] F. Bloch, Phys. Rev. 50 (1936) 259.

[116] J. S. Schwinger, Phys. Rev. 51 (1937) 544.

[117] O. Halpern and M. H. Johnson, Phys. Rev. 55 (1938) 898.

[118] L. Van Hove, Phys. Rev. 95 (1954) 249. 
[119] L. Van Hove, Phys. Rev. 95 (1954) 1374.

[120] Marshall W and Lovesey S W, Theory of Thermal Neutron Scattering, Oxford, Academic (1971).

[121] R. Balescu, Equilibrium and Nonequilibrium Statistical Mechanics, New York: Wiley (1975).

[122] J.-S. Caux, J. M. Maillet, Phys. Rev. Lett. 95 (2005) 077201.

[123] J.-S. Caux, R. Hagemans, J. M. Maillet, J. Stat. Mech. (2005) P09003.

[124] R. G. Pereira, J. Sirker, J.-S. Caux, R. Hagemans, J. M. Maillet, S. R. White, I. Affleck, Phys. Rev. Lett. 96 (2006) 257202.

[125] R. Hagemans, J.-S. Caux, J. M. Maillet, Proceedings of the "Tenth Training Course in the Physics of Correlated Electron Systems and High-Tc Superconductors", Salerno, Oct 2005, AIP Conference Proceedings 846 (2006) 245.

[126] R. G. Pereira, J. Sirker, J.-S. Caux, R. Hagemans, J. M. Maillet, S. R. White, I. Affleck, J. Stat. Mech. (2007) P08022.

[127] J. Sirker, R. G. Pereira, J.-S. Caux, R. Hagemans, J. M. Maillet, S. R. White, I. Affleck, Proceedings SCES '07, Houston, Physica B 403 (2008) 1520.

[128] J.-S. Caux, P. Calabrese and N. A. Slavnov, J. Stat. Mech. Theory Exp. (2007) P01008.

[129] D. Levy-Bencheton, G. Niccoli and V. Terras, Antiperiodic dynamical 6-vertex and periodic 8-vertex models: Form factors by separation of variables, to appear.

[130] F. Smirnov, J. Phys. A: Math. Gen. 31 (1998) 8953.

[131] O. Babelon, J. Phys. A 37 (2004) 303.

[132] A. B. Zamolodchikov, Int. J. Mod. Phys. A 3 (1988) 743.

[133] A. B. Zamolodchikov, Adv. Stud. Pure Math. 19 (1989) 641.

[134] Al. B. Zamolodchikov, Nucl. Phys. B 348 (1991) 619.

[135] R. Guida, N. Magnoli, Nucl. Phys. B 471 (1996) 361.

[136] M. A. Virasoro, Phys. Rev. D 1 (1970) 2933.

[137] A. A. Belavin, A. M. Polyakov, A. B. Zamolodchikov, Nucl. Phys. B 241 (1984) 333.

[138] P. Ginsparg, "Applied Conformal Field Theory", in: Fields, Strings and Critical Phenomena, Les Houches Lecture Notes 1988, eds. E. Brézin and J. Zinn-Justin, Elsevier, New York (1989).

[139] J. L. Cardy,Conformal Invariance and Statistical Mechanics, in Fields, Strings and Critical Phenomena, ed. E. Brézin and J. ZinnJustin, Les Houches (1988), Session XLIX North-Holland, Amsterdam (1990) 169.

[140] P. Di Francesco, P. Mathieu and D. Sénéchal, Conformal Field Theory, Springer, New York (1997).

[141] J. L. Cardy and G. Mussardo, Nucl. Phys. B 340 (1990) 387.

[142] F. A. Smirnov, Form Factors in Completely Integrable Models of Quantum Field Theory, World Scientific, (1992).

[143] A. Koubek, Nucl. Phys. B 435 (1995) 703.

[144] F. Smirnov, Nucl. Phys. B 453 (1995) 807.

[145] M. Jimbo, T. Miwa, Y. Takeyama, Counting minimal form factors of the restricted sine-Gordon model, arXiv:math-ph/0303059v6.

[146] G. Delfino and G. Niccoli, Nucl. Phys. B707 (2005) 381.

[147] G. Delfino and G. Niccoli, J. Stat. Mech. (2005) P04004

[148] G. Delfino and G. Niccoli, JHEP 05 (2006) 035.

[149] G. Delfino and G. Niccoli, Nucl. Phys. B 799 (2008) 364.

[150] A. B. Zamolodchikov, Pis. Zh. Eksp. Teor. Fiz. 25 (1977) 499; A. B. Zamolodchikov, Comm. Math. Phys. 55 (1977) 183 (Engl. transl.).

[151] A. B. Zamolodchikov, Al. B. Zamolodchikov, Ann. Phys. 120 (1979) 253.

[152] M. Karowski and H. J. Thun, Nucl. Phys. B 130 (1977) 295.

[153] V. E. Korepin, Comm. Math. Phys. 76 (1980) 165.

[154] G. Mussardo, Phys. Rep. 218 (1992) 215. 